\title{
An Approach to Increase Integration Rate of Elements of a Cascaded-inverter Circuit
}

\author{
E. L. Pankratov ${ }^{1,2}$ \\ ${ }^{1}$ Nizhny Novgorod State University, Russia \\ ${ }^{2}$ Nizhny Novgorod State Technical University, Russia \\ Received September 15, 2019; Revised October 21, 2019; Accepted October 28, 2019
}

Copyright $C 2019$ by authors, all rights reserved. Authors agree that this article remains permanently open access under the terms of the Creative Commons Attribution License 4.0 International License

\begin{abstract}
In this paper we introduce an approach to increase integration rate of elements of a cascaded-inverter. Framework the approach we consider a heterostructure with special configuration. Several specific areas of the heterostructure should be doped by diffusion or ion implantation. Annealing of dopant and/or radiation defects should be optimized.
\end{abstract}

Keywords Cascaded-inverter, Optimization of Manufacturing, Increasing of Element Integration Rate

\section{Introduction}

An actual and intensively solving problems of solid state electronics is increasing of integration rate of elements of integrated circuits ( $p$ - $n$-junctions, their systems et al) [1-8]. Increasing of the integration rate leads to necessity to decrease their dimensions. To decrease the dimensions are using several approaches. They are widely using laser and microwave types of annealing of infused dopants. These types of annealing are also widely using for annealing of radiation defects, generated during ion implantation [9-17]. Using the approaches gives a possibility to increase integration rate of elements of integrated circuits through inhomogeneity of technological parameters due to generating inhomogenous distribution of temperature. In this situation one can obtain decreasing dimensions of elements of integrated circuits [18] with account Arrhenius law $[1,3]$. Another approach to manufacture elements of integrated circuits with smaller dimensions is doping of heterostructure by diffusion or ion implantation [1-3]. However in this case optimization of dopant and/or radiation defects is required [18].
In this paper we consider a heterostructure. The heterostructure consist of a substrate and several epitaxial layers. Some sections have been manufactured in the epitaxial layers. Further we consider doping of these sections by diffusion or ion implantation. The doping gives a possibility to manufacture field-effect transistors framework a cascaded-inverter circuit so as it is shown on Figs. 1. The manufacturing leads to increasing density of elements of a cascaded-inverter circuit [4] due to presents of different materials in the heterostructure with different properties. It should be noted, that in the previous works manufacturing of integrated circuits and their elements in homogenous materials or some time in planar heterostructures. Using more simple structure in comparison with our case leads to smaller increasing of integration rate in comparison with processes in our heterostucture or do not leads to any increasing of the considered rate. After the above doping we consider optimized annealing of dopant and/or radiation defects. Optimization of annealing leads to manufacturing of more compact elements of integrated circuits and at the same time to increase of homogeneity of dopant distribution in doped area (in this situation one can find decreasing of local overheats during functioning of devices). Framework this paper we analyzed dynamics of redistribution of dopant and/or radiation defects during their annealing. The additional aim of this paper is development of analytical approach for modeling of technological process with account of nonlinearity of this process and variation of its parameters (diffusion coefficient of dopant and radiation, limit of solubility of dopant...). Using analytical approaches to model processes leads to decrease calculation time in comparison with numerical one. At the same time analytical approaches are more visible in comparison with numerical one. 


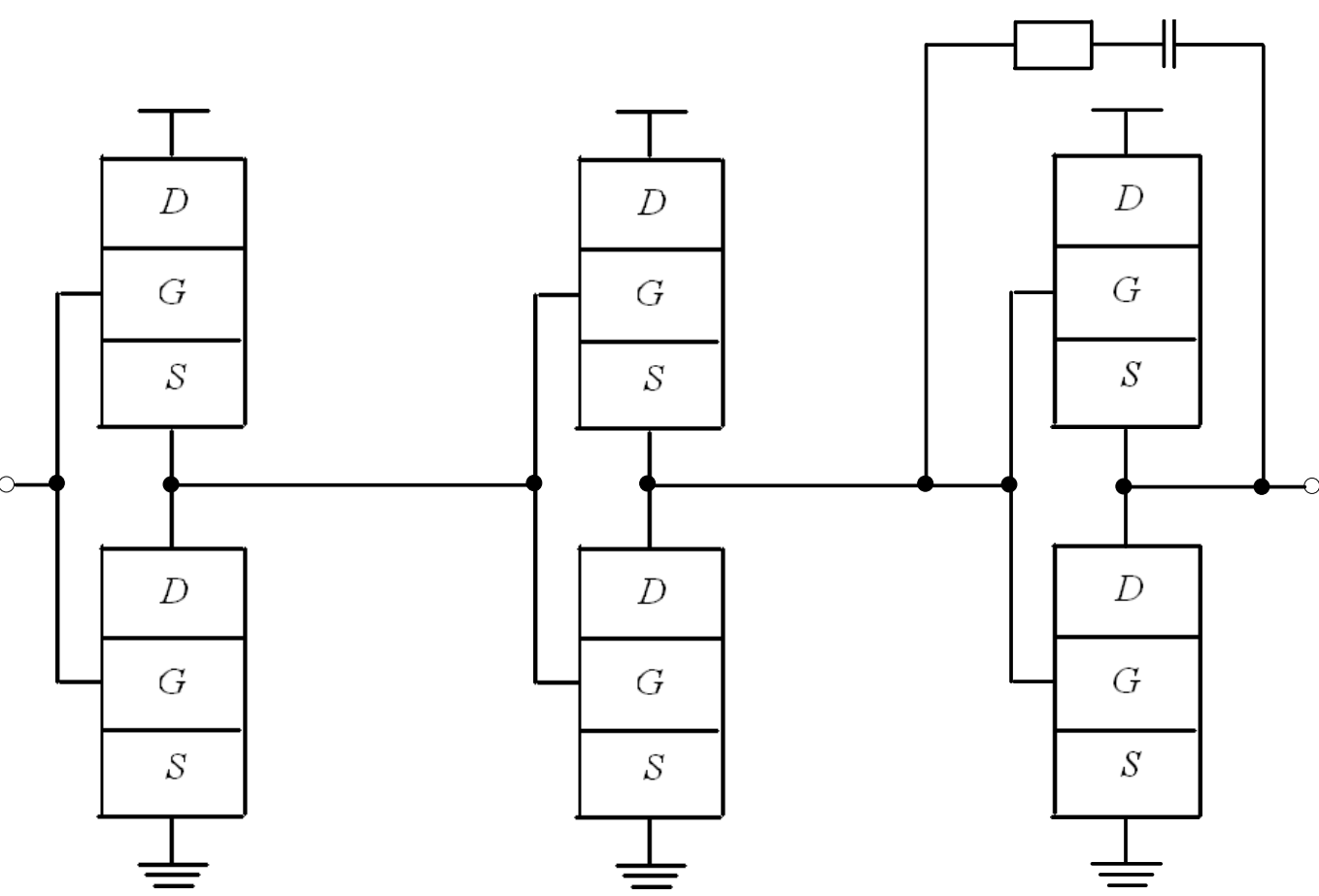

Figure 1. The considered cascaded-inverter [4]

\section{Method of Solution}

In this section we determine spatio-temporal distributions of concentrations of infused and implanted dopants. To determine these distributions we calculate appropriate solutions of the second Fick's law $[1,3,18]$

$$
\begin{gathered}
\frac{\partial C(x, y, z, t)}{\partial t}= \\
=\frac{\partial}{\partial x}\left[D_{C} \frac{\partial C(x, y, z, t)}{\partial x}\right]+\frac{\partial}{\partial y}\left[D_{C} \frac{\partial C(x, y, z, t)}{\partial y}\right]+\frac{\partial}{\partial z}\left[D_{C} \frac{\partial C(x, y, z, t)}{\partial z}\right] .
\end{gathered}
$$

Boundary and initial conditions for the equations are

$$
\begin{gathered}
\left.\frac{\partial C(x, y, z, t)}{\partial x}\right|_{x=0}=0,\left.\frac{\partial C(x, y, z, t)}{\partial x}\right|_{x=L_{x}}=0,\left.\frac{\partial C(x, y, z, t)}{\partial y}\right|_{y=0}=0,\left.\frac{\partial C(x, y, z, t)}{\partial y}\right|_{x=L_{y}}=0, \\
\left.\frac{\partial C(x, y, z, t)}{\partial z}\right|_{z=0}=0,\left.\frac{\partial C(x, y, z, t)}{\partial z}\right|_{x=L_{z}}=0, \mathrm{C}(\mathrm{x}, \mathrm{y}, \mathrm{z}, 0)=\mathrm{f}(\mathrm{x}, \mathrm{y}, \mathrm{z}) .
\end{gathered}
$$

The function $C(x, y, z, t)$ describes the spatio-temporal distribution of concentration of dopant; $T$ is the temperature of annealing; $D_{C}$ is the dopant diffusion coefficient. Value of dopant diffusion coefficient could be changed with changing materials of heterostructure, with changing temperature of materials (including annealing), with changing concentrations of dopant and radiation defects. We approximate dependences of dopant diffusion coefficient on parameters by the following relation with account results in Refs. [20-22]

$$
D_{C}=D_{L}(x, y, z, T)\left[1+\xi \frac{C^{\gamma}(x, y, z, t)}{P^{\gamma}(x, y, z, T)}\right]\left[1+\varsigma_{1} \frac{V(x, y, z, t)}{V^{*}}+\varsigma_{2} \frac{V^{2}(x, y, z, t)}{\left(V^{*}\right)^{2}}\right] \text {. }
$$


Here the function $D_{L}(x, y, z, T)$ describes the spatial (in heterostructure) and temperature (due to Arrhenius law) dependences of diffusion coefficient of dopant. The function $P(x, y, z, T)$ describes the limit of solubility of dopant. Parameter $\gamma \in[1,3]$ describes average quantity of charged defects interacted with atom of dopant [20]. The function $V$ $(x, y, z, t)$ describes the spatio-temporal distribution of concentration of radiation vacancies. Parameter $V^{*}$ describes the equilibrium distribution of concentration of vacancies. The considered concentrational dependence of dopant diffusion coefficient has been described in details in [20]. It should be noted, that using diffusion type of doping did not generation radiation defects. In this situation $\zeta_{1}=\zeta_{2}=0$. We determine spatio-temporal distributions of concentrations of radiation defects by solving the following system of equations $[21,22]$

$$
\begin{gathered}
\frac{\partial I(x, y, z, t)}{\partial t}=\frac{\partial}{\partial x}\left[D_{I}(x, y, z, T) \frac{\partial I(x, y, z, t)}{\partial x}\right]+\frac{\partial}{\partial y}\left[D_{I}(x, y, z, T) \frac{\partial I(x, y, z, t)}{\partial y}\right]+ \\
+\frac{\partial}{\partial z}\left[D_{I}(x, y, z, T) \frac{\partial I(x, y, z, t)}{\partial z}\right]-k_{I, V}(x, y, z, T) I(x, y, z, t) V(x, y, z, t)- \\
\frac{\partial V(x, y, z, t)}{\partial t}=\frac{\partial}{\partial x}\left[D_{V}(x, y, z, T) \frac{\partial V(x, y, z, t)}{\partial x}\right]+\frac{\partial}{\partial y}\left[D_{V}(x, y, z, T) \frac{\partial V(x, y, z, t)}{\partial y}\right]+ \\
+\frac{\partial}{\partial z}\left[D_{V}(x, y, z, T) \frac{\partial V(x, y, z, t)}{\partial z}\right]-k_{I, V}(x, y, z, T) I(x, y, z, t) V(x, y, z, t)+ \\
+k_{V, V}(x, y, z, T) V^{2}(x, y, z, t) .
\end{gathered}
$$

Boundary and initial conditions for these equations are

$$
\begin{gathered}
\left.\frac{\partial \rho(x, y, z, t)}{\partial x}\right|_{x=0}=0,\left.\frac{\partial \rho(x, y, z, t)}{\partial x}\right|_{x=L_{x}}=0,\left.\frac{\partial \rho(x, y, z, t)}{\partial y}\right|_{y=0}=0,\left.\frac{\partial \rho(x, y, z, t)}{\partial y}\right|_{y=L_{y}}=0, \\
\left.\frac{\partial \rho(x, y, z, t)}{\partial z}\right|_{z=0}=0,\left.\frac{\partial \rho(x, y, z, t)}{\partial z}\right|_{z=L_{z}}=0, \rho(\mathrm{x}, \mathrm{y}, \mathrm{z}, 0)=\mathrm{f}_{\rho}(\mathrm{x}, \mathrm{y}, \mathrm{z}) .
\end{gathered}
$$

Here $\rho=I, V$. The function $I(x, y, z, t)$ describes the spatio-temporal distribution of concentration of radiation interstitials; $D_{\rho}(x, y, z, T)$ are the diffusion coefficients of point radiation defects; terms $V^{2}(x, y, z, t)$ and $I^{2}(x, y, z, t)$ correspond to generation divacancies and diinterstitials; $k_{I, V}(x, y, z, T)$ is the parameter of recombination of point radiation defects; $k_{I, I}(x, y, z, T)$ and $k_{V, V}(x, y, z, T)$ are the parameters of generation of simplest complexes of point radiation defects.

Further we determine distributions in space and time of concentrations of divacancies $\Phi_{V}(x, y, z, t)$ and diinterstitials $\Phi_{I}(x, y, z, t)$ by solving the following system of equations $[21,22]$

$$
\begin{aligned}
& \frac{\partial \Phi_{I}(x, y, z, t)}{\partial t}=\frac{\partial}{\partial x}\left[D_{\Phi I}(x, y, z, T) \frac{\partial \Phi_{I}(x, y, z, t)}{\partial x}\right]+\frac{\partial}{\partial y}\left[D_{\Phi I}(x, y, z, T) \frac{\partial \Phi_{I}(x, y, z, t)}{\partial y}\right]+ \\
& +\frac{\partial}{\partial z}\left[D_{\Phi I}(x, y, z, T) \frac{\partial \Phi_{I}(x, y, z, t)}{\partial z}\right]+k_{I, I}(x, y, z, T) I^{2}(x, y, z, t)-k_{I}(x, y, z, T) I(x, y, z, t) \\
& \frac{\partial \Phi_{V}(x, y, z, t)}{\partial t}=\frac{\partial}{\partial x}\left[D_{\Phi V}(x, y, z, T) \frac{\partial \Phi_{V}(x, y, z, t)}{\partial x}\right]+\frac{\partial}{\partial y}\left[D_{\Phi V}(x, y, z, T) \frac{\partial \Phi_{V}(x, y, z, t)}{\partial y}\right]+
\end{aligned}
$$




$$
+\frac{\partial}{\partial z}\left[D_{\Phi V}(x, y, z, T) \frac{\partial \Phi_{V}(x, y, z, t)}{\partial z}\right]+k_{V, V}(x, y, z, T) V^{2}(x, y, z, t)-k_{V}(x, y, z, T) V(x, y, z, t) .
$$

Boundary and initial conditions for these equations are

$$
\begin{gathered}
\left.\frac{\partial \Phi_{\rho}(x, y, z, t)}{\partial x}\right|_{x=0}=0,\left.\frac{\partial \Phi_{\rho}(x, y, z, t)}{\partial x}\right|_{x=L_{x}}=0,\left.\frac{\partial \Phi_{\rho}(x, y, z, t)}{\partial y}\right|_{y=0}=0,\left.\frac{\partial \Phi_{\rho}(x, y, z, t)}{\partial y}\right|_{y=L_{y}}=0 \\
\left.\frac{\partial \Phi_{\rho}(x, y, z, t)}{\partial z}\right|_{z=0}=0,\left.\frac{\partial \Phi_{\rho}(x, y, z, t)}{\partial z}\right|_{z=L_{z}}=0 \\
\Phi_{\mathrm{I}}(\mathrm{x}, \mathrm{y}, \mathrm{z}, 0)=\mathrm{f}_{\Phi \mathrm{I}}(\mathrm{x}, \mathrm{y}, \mathrm{z}), \Phi_{\mathrm{V}}(\mathrm{x}, \mathrm{y}, \mathrm{z}, 0)=\mathrm{f}_{\Phi \mathrm{V}}(\mathrm{x}, \mathrm{y}, \mathrm{z}) .
\end{gathered}
$$

Here $D_{\Phi \rho}(x, y, z, T)$ are the diffusion coefficients of the above complexes of radiation defects; $k_{l}(x, y, z, T)$ and $k_{V}(x, y, z, T)$ are the parameters of decay of these complexes.

We calculate distributions of concentrations of point radiation defects in space and time by recently elaborated approach [18]. The approach based on transformation of approximations of diffusion coefficients in the following form: $D_{\rho}(x, y, z, T)=D_{0 \rho}\left[1+\varepsilon_{\rho} g_{\rho}(x, y, z, T)\right]$, where $D_{0 \rho}$ are the average values of diffusion coefficients, $0 \leq \varepsilon_{\rho}<1,\left|g_{\rho}(x, y, z, T)\right| \leq 1, \rho$ $=I, V$. We also used analogous transformation of approximations of parameters of recombination of point defects and parameters of generation of their complexes: $k_{I, V}(x, y, z, T)=k_{0 I, V}\left[1+\varepsilon_{I, V} g_{I, V}(x, y, z, T)\right], k_{I, I}(x, y, z, T)=k_{0 I, I}\left[1+\varepsilon_{I, I} g_{I, I}(x, y, z, T)\right]$ and $k_{V, V}(x, y, z, T)=k_{0 V, V}\left[1+\varepsilon_{V, V} g_{V, V}(x, y, z, T)\right]$, where $k_{0 \rho 1, \rho 2}$ are the their average values, $0 \leq \varepsilon_{I, V}<1,0 \leq \varepsilon_{I, I}<1,0 \leq \varepsilon_{V, V}<1, \mid$ $g_{I, V}(x, y, z, T)|\leq 1, \quad| \quad g_{I, I}(x, y, z, T)|\leq 1, \quad| g_{V, V}(x, y, z, T) \mid \leq 1 . \quad$ Let us introduce the following dimensionless variables: $\widetilde{I}(x, y, z, t)=I(x, y, z, t) / I^{*}, \widetilde{V}(x, y, z, t)==V(x, y, z, t) / V^{*}, \omega=L^{2} k_{0 I, V} / \sqrt{D_{0 I} D_{0 V}}$, $\Omega_{\rho}=L^{2} k_{0 \rho, \rho} / \sqrt{D_{0 I} D_{0 V}}, \vartheta=\sqrt{D_{0 I} D_{0 V}} t / L^{2}, \chi=x / L_{x}, \eta=y / L_{y}, \phi=z / L_{z}$. The introduction leads to transformation of Eqs.(4) and conditions (5) to the following form

$$
\begin{aligned}
& \frac{\partial \tilde{I}(\chi, \eta, \phi, \vartheta)}{\partial \vartheta}=\frac{D_{0 I}}{\sqrt{D_{0 I} D_{0 V}}} \frac{\partial}{\partial \chi}\left\{\left[1+\varepsilon_{I} g_{I}(\chi, \eta, \phi, T)\right] \frac{\partial \tilde{I}(\chi, \eta, \phi, \vartheta)}{\partial \chi}\right\}+\frac{\partial}{\partial \eta}\left\{\left[1+\varepsilon_{I} g_{I}(\chi, \eta, \phi, T)\right] \times\right. \\
& \left.\times \frac{\partial \widetilde{I}(\chi, \eta, \phi, \vartheta)}{\partial \eta}\right\} \frac{D_{0 I}}{\sqrt{D_{0 I} D_{0 V}}}+\frac{D_{0 I}}{\sqrt{D_{0 I} D_{0 V}}} \frac{\partial}{\partial \phi}\left\{\left[1+\varepsilon_{I} g_{I}(\chi, \eta, \phi, T)\right] \frac{\partial \widetilde{I}(\chi, \eta, \phi, \vartheta)}{\partial \phi}\right\}-\widetilde{I}(\chi, \eta, \phi, \vartheta) \times \\
& \times \omega\left[1+\varepsilon_{I, V} g_{I, V}(\chi, \eta, \phi, T)\right] \widetilde{V}(\chi, \eta, \phi, \vartheta)-\Omega_{I}\left[1+\varepsilon_{I, I} g_{I, I}(\chi, \eta, \phi, T)\right] \widetilde{I}^{2}(\chi, \eta, \phi, \vartheta) \\
& \frac{\partial \widetilde{V}(\chi, \eta, \phi, \vartheta)}{\partial \vartheta}=\frac{D_{0 V}}{\sqrt{D_{0 I} D_{0 V}}} \frac{\partial}{\partial \chi}\left\{\left[1+\varepsilon_{V} g_{V}(\chi, \eta, \phi, T)\right] \frac{\partial \tilde{V}(\chi, \eta, \phi, \vartheta)}{\partial \chi}\right\}+\frac{\partial}{\partial \eta}\left\{\left[1+\varepsilon_{V} g_{V}(\chi, \eta, \phi, T)\right] \times\right. \\
& \left.\times \frac{\partial \widetilde{V}(\chi, \eta, \phi, \vartheta)}{\partial \eta}\right\} \frac{D_{0 V}}{\sqrt{D_{0 I} D_{0 V}}}+\frac{D_{0 V}}{\sqrt{D_{0 I} D_{0 V}}} \frac{\partial}{\partial \phi}\left\{\left[1+\varepsilon_{V} g_{V}(\chi, \eta, \phi, T)\right] \frac{\partial \widetilde{V}(\chi, \eta, \phi, \vartheta)}{\partial \phi}\right\}-\widetilde{I}(\chi, \eta, \phi, \vartheta) \times \\
& \times \omega\left[1+\varepsilon_{I, V} g_{I, V}(\chi, \eta, \phi, T)\right] \widetilde{V}(\chi, \eta, \phi, \vartheta)-\Omega_{V}\left[1+\varepsilon_{V, V} g_{V, V}(\chi, \eta, \phi, T)\right] \widetilde{V}^{2}(\chi, \eta, \phi, \vartheta) \\
& \left.\frac{\partial \widetilde{\rho}(\chi, \eta, \phi, \vartheta)}{\partial \chi}\right|_{\chi=0}=0,\left.\frac{\partial \widetilde{\rho}(\chi, \eta, \phi, \vartheta)}{\partial \chi}\right|_{\chi=1}=0,\left.\frac{\partial \widetilde{\rho}(\chi, \eta, \phi, \vartheta)}{\partial \eta}\right|_{\eta=0}=0,\left.\frac{\partial \widetilde{\rho}(\chi, \eta, \phi, \vartheta)}{\partial \eta}\right|_{\eta=1}=0 \text {, }
\end{aligned}
$$




$$
\left.\frac{\partial \widetilde{\rho}(\chi, \eta, \phi, \vartheta)}{\partial \phi}\right|_{\phi=0}=0,\left.\frac{\partial \widetilde{\rho}(\chi, \eta, \phi, \vartheta)}{\partial \phi}\right|_{\phi=1}=0, \widetilde{\rho}(\chi, \eta, \phi, \vartheta)=\frac{f_{\rho}(\chi, \eta, \phi, \vartheta)}{\rho^{*}} .
$$

We determine solutions of Eqs.(8) with conditions (9) framework recently introduced approach [18], i.e. as the power series

$$
\widetilde{\rho}(\chi, \eta, \phi, \vartheta)=\sum_{i=0}^{\infty} \varepsilon_{\rho}^{i} \sum_{j=0}^{\infty} \omega^{j} \sum_{k=0}^{\infty} \Omega_{\rho}^{k} \widetilde{\rho}_{i j k}(\chi, \eta, \phi, \vartheta) .
$$

Substitution of the series (10) into Eqs.(8) and conditions (9) gives us possibility to obtain equations for initial-order approximations of concentration of point defects $\tilde{I}_{000}(\chi, \eta, \phi, \vartheta)$ and $\widetilde{V}_{000}(\chi, \eta, \phi, \vartheta)$ and corrections for them $\widetilde{I}_{i j k}(\chi, \eta, \phi, \vartheta)$ and $\widetilde{V}_{i j k}(\chi, \eta, \phi, \vartheta), i \geq 1, j \geq 1, k \geq 1$. The equations are presented in the Appendix. Solutions of the equations could be obtained by standard Fourier approach $[24,25]$. The solutions are presented in the Appendix.

Now we calculate distributions of concentrations of simplest complexes of point radiation defects in space and time. To determine the distributions we transform approximations of diffusion coefficients in the following form: $D_{\Phi \rho}(x, y, z, T)=D_{0 \Phi_{\rho}}\left[1+\varepsilon_{\Phi \rho} g_{\Phi \rho}(x, y, z, T)\right]$, where $D_{0 \Phi_{\rho}}$ are the average values of diffusion coefficients. In this situation the Eqs.(6) could be written as

$$
\begin{aligned}
& \frac{\partial \Phi_{I}(x, y, z, t)}{\partial t}=D_{0 \Phi I} \frac{\partial}{\partial x}\left\{\left[1+\varepsilon_{\Phi I} g_{\Phi I}(x, y, z, T)\right] \frac{\partial \Phi_{I}(x, y, z, t)}{\partial x}\right\}+k_{I, I}(x, y, z, T) I^{2}(x, y, z, t)+ \\
& +D_{0 \oplus I} \frac{\partial}{\partial y}\left\{\left[1+\varepsilon_{\Phi I} g_{\phi I}(x, y, z, T)\right] \frac{\partial \Phi_{I}(x, y, z, t)}{\partial y}\right\}+D_{0 \Phi I} \frac{\partial}{\partial z}\left\{\left[1+\varepsilon_{\Phi I} g_{\Phi I}(x, y, z, T)\right] \frac{\partial \Phi_{I}(x, y, z, t)}{\partial z}\right\}- \\
& -k_{I}(x, y, z, T) I(x, y, z, t) \\
& \frac{\partial \Phi_{V}(x, y, z, t)}{\partial t}=D_{0 \Phi V} \frac{\partial}{\partial x}\left\{\left[1+\varepsilon_{\Phi V} g_{\Phi V}(x, y, z, T)\right] \frac{\partial \Phi_{V}(x, y, z, t)}{\partial x}\right\}+k_{I, I}(x, y, z, T) I^{2}(x, y, z, t)+ \\
& +D_{0 \Phi V} \frac{\partial}{\partial y}\left\{\left[1+\varepsilon_{\Phi V} g_{\Phi V}(x, y, z, T)\right] \frac{\partial \Phi_{V}(x, y, z, t)}{\partial y}\right\}+D_{0 \Phi V} \frac{\partial}{\partial z}\left\{\left[1+\varepsilon_{\Phi V} g_{\Phi V}(x, y, z, T)\right] \frac{\partial \Phi_{V}(x, y, z, t)}{\partial z}\right\}- \\
& -k_{I}(x, y, z, T) I(x, y, z, t) \text {. }
\end{aligned}
$$

Farther we determine solutions of above equations as the following power series

$$
\Phi_{\rho}(x, y, z, t)=\sum_{i=0}^{\infty} \varepsilon_{\Phi \rho}^{i} \Phi_{\rho i}(x, y, z, t) .
$$


Now we used the series (11) into Eqs.(6) and appropriate boundary and initial conditions. The using gives the possibility to obtain equations for initial-order approximations of concentrations of complexes of defects $\Phi_{\rho 0}(x, y, z, t)$, corrections for them $\Phi_{\rho i}(x, y, z, t)$ (for them $i \geq 1$ ) and boundary and initial conditions for them. We remove equations and conditions to the Appendix. Solutions of the equations have been calculated by standard approaches $[24,25]$ and presented in the Appendix.

Now we calculate distribution of concentration of dopant in space and time by using the approach, which was used for analysis of radiation defects. To use the approach we consider following transformation of approximation of dopant diffusion coefficient: $D_{L}(x, y, z, T)=D_{0 L}[1+$ $\left.\varepsilon_{L} g_{L}(x, y, z, T)\right]$, where $D_{0 L}$ is the average value of dopant diffusion coefficient, $0 \leq \varepsilon_{L}<1,\left|g_{L}(x, y, z, T)\right| \leq 1$. Farther we consider solution of Eq. (1) as the following series:

$$
C(x, y, z, t)=\sum_{i=0}^{\infty} \varepsilon_{L}^{i} \sum_{j=1}^{\infty} \xi^{j} C_{i j}(x, y, z, t) .
$$

Using the relation into Eq.(1) and conditions (2) leads to obtaining equations for the functions $C_{i j}(x, y, z, t)(i \geq 1, j \geq 1)$, boundary and initial conditions for them. The equations are presented in the Appendix. Solutions of the equations have been calculated by standard approaches (see, for example, $[24,25])$. The solutions are presented in the Appendix.

We analyzed distributions of concentrations of dopant and radiation defects in space and time analytically by using the second-order approximations on all parameters, which have been used in appropriate series. Usually the second-order approximations are enough good approximations to make qualitative analysis and to obtain quantitative results. All analytical results have been checked by numerical simulation.

\section{Discussion}

In this section we analyzed spatio-temporal distributions of concentrations of dopants. Figs. 2 shows typical spatial distributions of concentrations of dopants in neighborhood of interfaces of heterostructures. We calculate these distributions of concentrations of dopants under the following condition: value of dopant diffusion coefficient in doped area is larger, than value of dopant diffusion coefficient in nearest areas. One can find from Figs. 2 increasing of compactness of dopant distributions in doped area with increasing of homogeneity of distribution of concentration of dopant at one time. In this situation one can obtain increasing of density of field-effect transistors framework considered circuit and at one time decreasing local overheats during functioning of devices in doped areas. It should be noted, that transistors and $p-n$-junctions usually manufacturing in homogenous materials with appropriate wide distributions of dopant (curves 1 on Figs. 2) [1-8]. In this situation one can find devices with larger dimensions and more spread boundaries. Changing relation between values of dopant diffusion coefficients leads to opposite result (see Figs. 3).

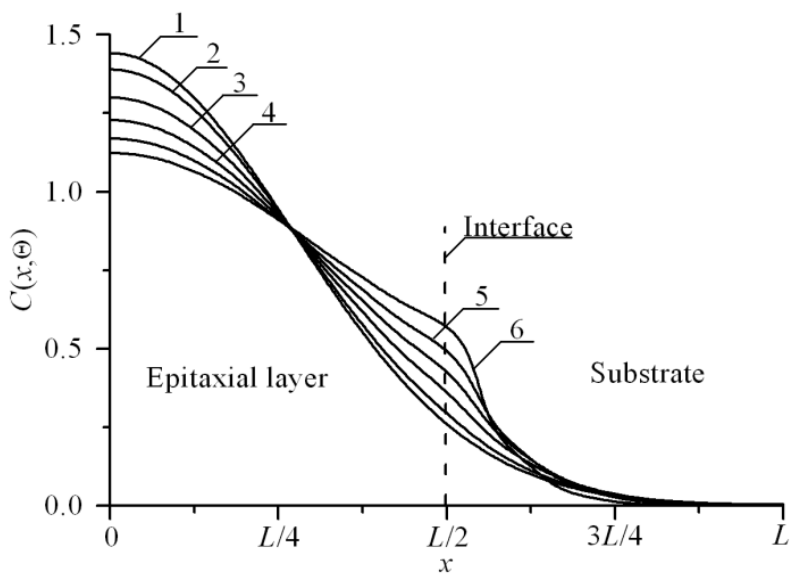

Figure 2a. Dependences of concentration of dopant, infused in heterostructure from Figs. 1, on coordinate in direction, which is perpendicular to interface between epitaxial layer substrate. Difference between values of dopant diffusion coefficient in layers of heterostructure increases with increasing of number of curves. Value of dopant diffusion coefficient in the epitaxial layer is larger, than value of dopant diffusion coefficient in the substrate

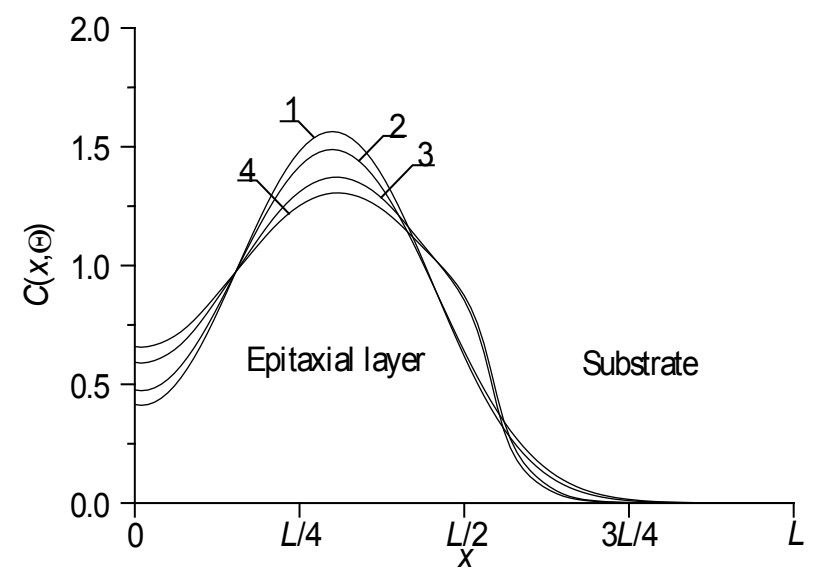

Figure $2 \boldsymbol{b}$. Dependences of concentration of dopant, implanted in heterostructure from Figs. 1, on coordinate in direction, which is perpendicular to interface between epitaxial layer substrate. Difference between values of dopant diffusion coefficient in layers of heterostructure increases with increasing of number of curves. Value of dopant diffusion coefficient in the epitaxial layer is larger, than value of dopant diffusion coefficient in the substrate. Curve 1 corresponds to homogenous sample and annealing time $\Theta=0.0048\left(L_{x}{ }^{2}+L_{y}{ }^{2}+L_{z}{ }^{2}\right) / D_{0}$. Curve 2 corresponds to homogenous sample and annealing time $\Theta=0.0057\left(L_{x}{ }^{2}+L_{y}{ }^{2}+L_{z}{ }^{2}\right) / D_{0}$. Curves 3 and 4 correspond to heterostructure from Figs. 1; annealing times $\Theta=0.0048\left(L_{x}{ }^{2}+L_{y}{ }^{2}+L_{z}{ }^{2}\right) / D_{0}$ and $\Theta=0.0057\left(L_{x}{ }^{2}+L_{y}{ }^{2}+L_{z}{ }^{2}\right) / D_{0}$, respectively 


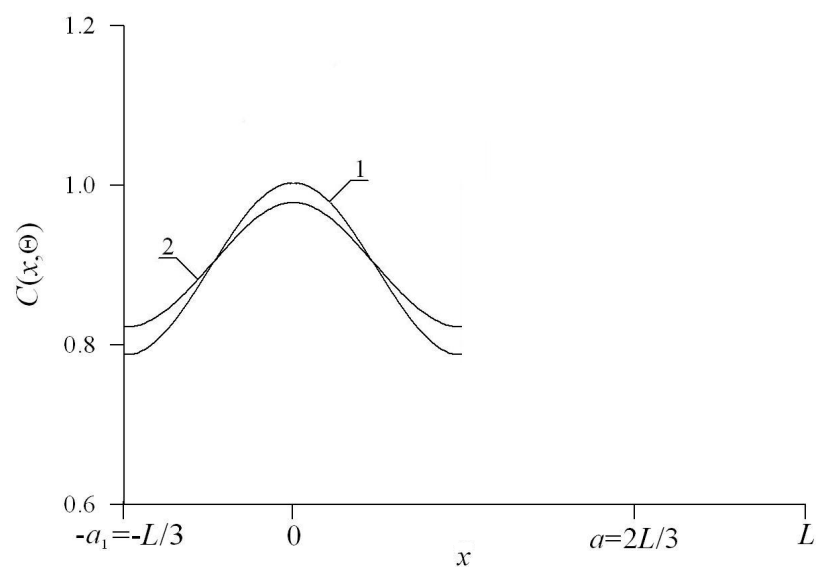

Figure 3a. Distributions of concentration of dopant, infused in average section of epitaxial layer of heterostructure from Figs. 1 in direction parallel to interface between epitaxial layer and substrate of heterostructure. Difference between values of dopant diffusion coefficients increases with increasing of number of curves. Value of dopant diffusion coefficient in this section is smaller, than value of dopant diffusion coefficient in nearest sections

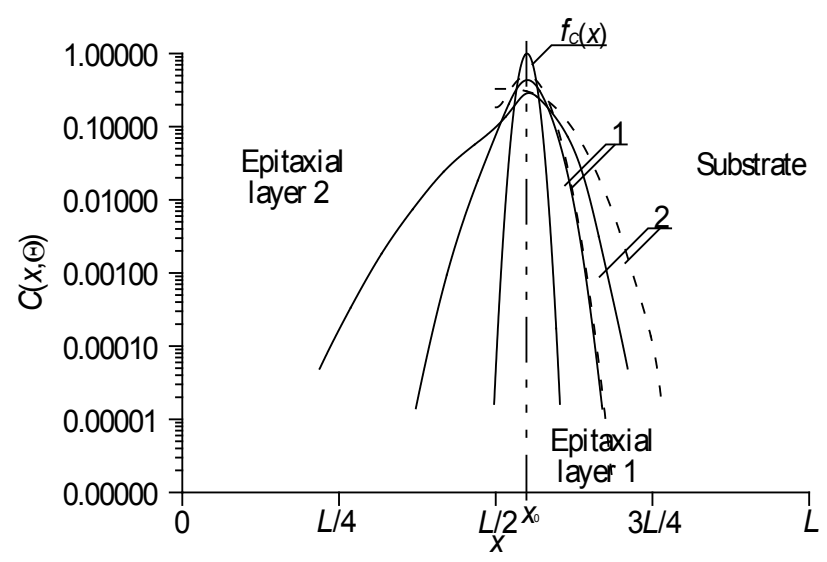

Figure 3b. Calculated distributions of implanted dopant in epitaxial layers of heterostructure. Solid lines are spatial distributions of implanted dopant in system of two epitaxial layers. Dushed lines are spatial distributions of implanted dopant in one epitaxial layer. Annealing time increases with increasing of number of curves

It should be noted, that framework the considered approach one shall optimize annealing of dopant and/or radiation defects. Reason for this optimization is following. If annealing of dopant is short, than dopant has no time to achieve interface between materials of heterostructure and modification of distribution of dopant due to the interface could not be obtained. In this situation one can find recently introduced technological schemes [1-8]. If annealing time is very large, than distribution of dopant will be too homogenous. In this situation interface between doped and undoped areas will be almost absent. In this situation it should be used compromise value of annealing time. This choosing was absents in related works and became original procedure of author of this paper. To make this choosing we use recently introduced criterion [26-34]. The choosing based on approximation of real distribution of concentration of dopant by step-wise function $\psi(x, y, z)$ (see Figs. 4). Farther the required values of optimal annealing time have been calculated by minimization the following mean-squared error

$$
U=\frac{1}{L_{x} L_{y} L_{z}} \int_{0}^{L_{x}} \int_{0}^{L_{y} L_{z}} \int_{0}[C(x, y, z, \Theta)-\psi(x, y, z)] d z d y d x
$$

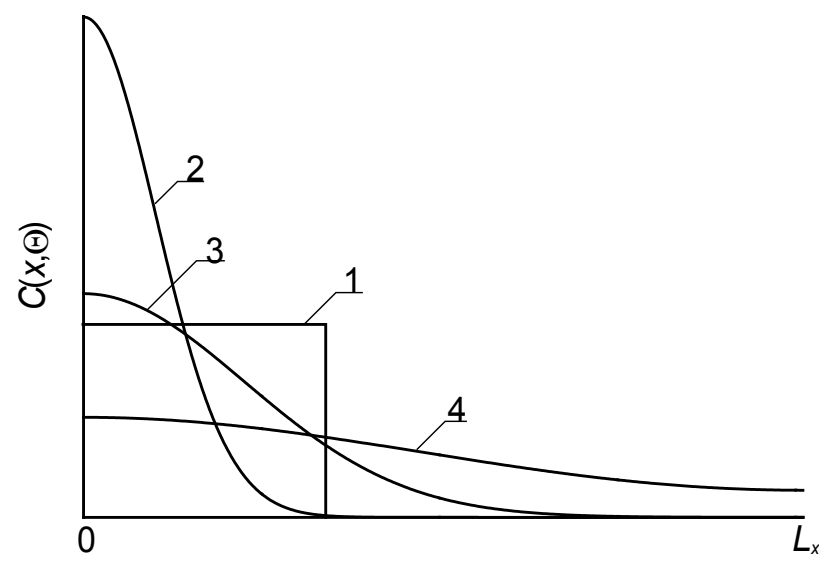

Figure 4a. Distributions of concentration of infused dopant in depth of heterostructure from Fig. 1 for different values of annealing time (curves 2-4) and idealized step-wise approximation (curve 1). Increasing of number of curve corresponds to increasing of annealing time

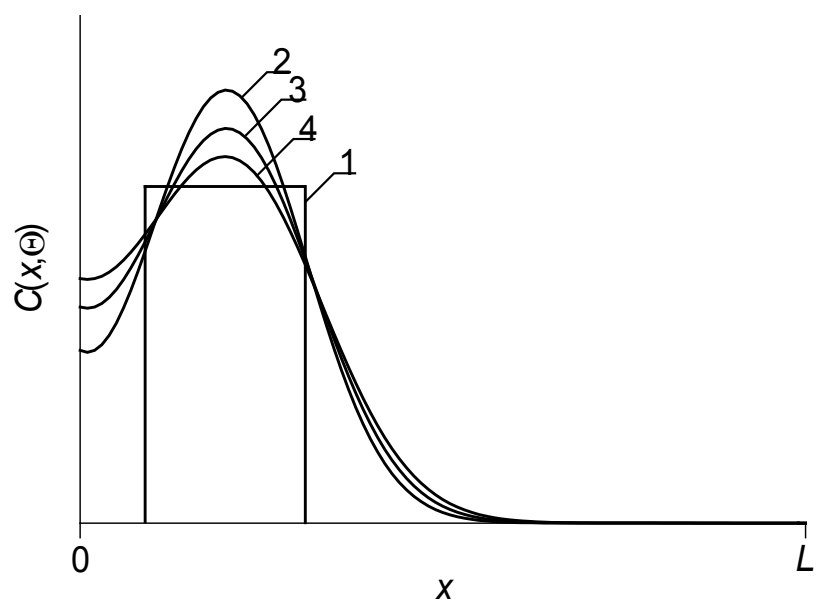

Figure 4b. Distributions of concentration of implanted dopant in depth of heterostructure from Fig. 1 for different values of annealing time (curves 2-4) and idealized step-wise approximation (curve 1). Increasing of number of curve corresponds to increasing of annealing time

We show optimal values of annealing time as functions of several parameters on Figs. 5. It is known, that standard step of manufactured ion-doped structures is annealing of radiation defects. In the ideal case after finishing this annealing dopant achieves interface between layers of heterostructure. If the dopant has no enough time to achieve the interface, it is practicably to anneal the dopant additionally. The Fig. $5 b$ shows the described dependences of optimal values of additional annealing time for the same parameters as for Fig. $5 a$. Necessity to anneal radiation defects leads to smaller values of optimal annealing of implanted dopant in comparison with optimal annealing time of infused dopant. 


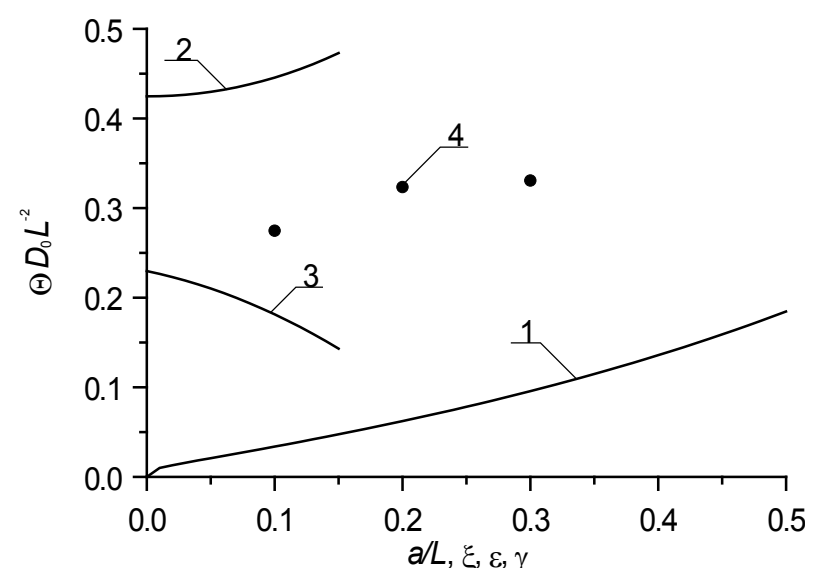

Figure 5a. Dimensionless optimal annealing time of infused dopant as a function of several parameters. Curve 1 describes the dependence of the annealing time on the relation $a / L$ and $\xi=\gamma=0$ for equal to each other values of dopant diffusion coefficient in all parts of heterostructure. Curve 2 describes the dependence of the annealing time on value of parameter $\varepsilon$ for $a / L=1 / 2$ and $\xi=\gamma=0$. Curve 3 describes the dependence of the annealing time on value of parameter $\xi$ for $a / L=1 / 2$ and $\varepsilon=\gamma=0$. Curve 4 describes the dependence of the annealing time on value of parameter $\gamma$ for $a / L=1 / 2$ and $\varepsilon=\xi=0$

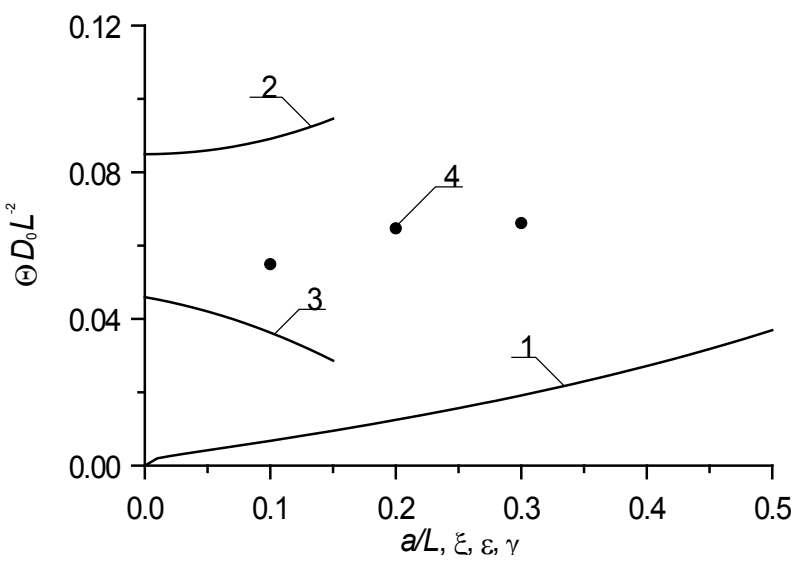

Figure 5b. Dimensionless optimal annealing time of implanted dopant as a function of several parameters. Curve 1 describes the dependence of the annealing time on the relation $a / L$ and $\xi=\gamma=0$ for equal to each other values of dopant diffusion coefficient in all parts of heterostructure. Curve 2 describes the dependence of the annealing time on value of parameter $\varepsilon$ for $a / L=1 / 2$ and $\xi=\gamma=0$. Curve 3 describes the dependence of the annealing time on value of parameter $\xi$ for $a / L=1 / 2$ and $\varepsilon=\gamma=0$. Curve 4 describes the dependence of the annealing time on value of parameter $\gamma$ for $a / L=1 / 2$ and $\varepsilon=\xi=0$

\section{Conclusions}

In this paper we introduce an approach to increase integration rate of element of a cascaded-inverter circuit. The approach gives us possibility to decrease area of the elements with decreasing of the element's thickness. At the same time we introduce an analytical approach to model technological process with account nonlinearity of this process and variation of different technological processes.

\section{Appendix}

Equations for the functions $\widetilde{I}_{i j k}(\chi, \eta, \phi, \vartheta)$ and $\widetilde{V}_{i j k}(\chi, \eta, \phi, \vartheta), i \geq 0, j \geq 0, k \geq 0$ and conditions for them

$$
\begin{aligned}
& \frac{\partial \widetilde{I}_{000}(\chi, \eta, \phi, \vartheta)}{\partial \vartheta}=\sqrt{\frac{D_{0 I}}{D_{0 V}}}\left[\frac{\partial^{2} \widetilde{I}_{000}(\chi, \eta, \phi, \vartheta)}{\partial \chi^{2}}+\frac{\partial^{2} \widetilde{I}_{000}(\chi, \eta, \phi, \vartheta)}{\partial \eta^{2}}+\frac{\partial^{2} \widetilde{I}_{000}(\chi, \eta, \phi, \vartheta)}{\partial \phi^{2}}\right] \\
& \frac{\partial \widetilde{V}_{000}(\chi, \eta, \phi, \vartheta)}{\partial \vartheta}=\sqrt{\frac{D_{0 V}}{D_{0 I}}}\left[\frac{\partial^{2} \widetilde{V}_{000}(\chi, \eta, \phi, \vartheta)}{\partial \chi^{2}}+\frac{\partial^{2} \widetilde{V}_{000}(\chi, \eta, \phi, \vartheta)}{\partial \eta^{2}}+\frac{\partial^{2} \widetilde{V}_{000}(\chi, \eta, \phi, \vartheta)}{\partial \phi^{2}}\right] ; \\
& \frac{\partial \widetilde{I}_{i 00}(\chi, \vartheta)}{\partial \vartheta}=\sqrt{\frac{D_{0 I}}{D_{0 V}}}\left[\frac{\partial^{2} \widetilde{I}_{i 00}(\chi, \eta, \phi, \vartheta)}{\partial \chi^{2}}+\frac{\partial^{2} \widetilde{I}_{i 00}(\chi, \eta, \phi, \vartheta)}{\partial \eta^{2}}+\frac{\partial^{2} \widetilde{I}_{i 00}(\chi, \eta, \phi, \vartheta)}{\partial \phi^{2}}\right]+\sqrt{\frac{D_{0 I}}{D_{0 V}}} \times \\
& \times\left\{\frac{\partial}{\partial \chi}\left[g_{I}(\chi, \eta, \phi, T) \frac{\partial \widetilde{I}_{i-100}(\chi, \eta, \phi, \vartheta)}{\partial \chi}\right]+\frac{\partial}{\partial \eta}\left[g_{I}(\chi, \eta, \phi, T) \frac{\partial \widetilde{I}_{i-100}(\chi, \eta, \phi, \vartheta)}{\partial \eta}\right]+\right. \\
& \left.+\frac{\partial}{\partial \phi}\left[g_{I}(\chi, \eta, \phi, T) \frac{\partial \widetilde{I}_{i-100}(\chi, \eta, \phi, \vartheta)}{\partial \phi}\right]\right\}, \mathrm{i} \geq 1,
\end{aligned}
$$




$$
\begin{gathered}
\frac{\partial \widetilde{V}_{i 00}(\chi, \vartheta)}{\partial \vartheta}=\sqrt{\frac{D_{0 V}}{D_{0 I}}}\left[\frac{\partial^{2} \widetilde{V}_{i 00}(\chi, \eta, \phi, \vartheta)}{\partial \chi^{2}}+\frac{\partial^{2} \widetilde{V}_{i 00}(\chi, \eta, \phi, \vartheta)}{\partial \eta^{2}}+\frac{\partial^{2} \widetilde{V}_{i 00}(\chi, \eta, \phi, \vartheta)}{\partial \phi^{2}}\right]+\sqrt{\frac{D_{0 V}}{D_{0 I}}} \times \\
\times \frac{\partial}{\partial \chi}\left[g_{V}(\chi, \eta, \phi, T) \frac{\partial \widetilde{V}_{i-100}(\chi, \eta, \phi, \vartheta)}{\partial \chi}\right]+\sqrt{\frac{D_{0 V}}{D_{0 I}}} \frac{\partial}{\partial \eta}\left[g_{V}(\chi, \eta, \phi, T) \frac{\partial \widetilde{V}_{i-100}(\chi, \eta, \phi, \vartheta)}{\partial \eta}\right]+ \\
+\sqrt{\frac{D_{0 V}}{D_{0 I}}} \frac{\partial}{\partial \phi}\left[g_{V}(\chi, \eta, \phi, T) \frac{\partial \widetilde{V}_{i-100}(\chi, \eta, \phi, \vartheta)}{\partial \phi}\right], \mathrm{i} \geq 1,
\end{gathered}
$$$$
\frac{\partial \widetilde{I}_{010}(\chi, \eta, \phi, \vartheta)}{\partial \vartheta}=\sqrt{\frac{D_{0 I}}{D_{0 V}}}\left[\frac{\partial^{2} \widetilde{I}_{010}(\chi, \eta, \phi, \vartheta)}{\partial \chi^{2}}+\frac{\partial^{2} \widetilde{I}_{010}(\chi, \eta, \phi, \vartheta)}{\partial \eta^{2}}+\frac{\partial^{2} \widetilde{I}_{010}(\chi, \eta, \phi, \vartheta)}{\partial \phi^{2}}\right]-
$$$$
-\left[1+\varepsilon_{I, V} g_{I, V}(\chi, \eta, \phi, T)\right] \widetilde{I}_{000}(\chi, \eta, \phi, \vartheta) \widetilde{V}_{000}(\chi, \eta, \phi, \vartheta)
$$$$
\frac{\partial \widetilde{V}_{010}(\chi, \eta, \phi, \vartheta)}{\partial \vartheta}=\sqrt{\frac{D_{0 V}}{D_{0 I}}}\left[\frac{\partial^{2} \widetilde{V}_{010}(\chi, \eta, \phi, \vartheta)}{\partial \chi^{2}}+\frac{\partial^{2} \widetilde{V}_{010}(\chi, \eta, \phi, \vartheta)}{\partial \eta^{2}}+\frac{\partial^{2} \widetilde{V}_{010}(\chi, \eta, \phi, \vartheta)}{\partial \phi^{2}}\right]-
$$$$
-\left[1+\varepsilon_{I, V} g_{I, V}(\chi, \eta, \phi, T)\right] \widetilde{I}_{000}(\chi, \eta, \phi, \vartheta) \widetilde{V}_{000}(\chi, \eta, \phi, \vartheta) ;
$$$$
\frac{\partial \widetilde{I}_{020}(\chi, \eta, \phi, \vartheta)}{\partial \vartheta}=\sqrt{\frac{D_{0 I}}{D_{0 V}}}\left[\frac{\partial^{2} \widetilde{I}_{020}(\chi, \eta, \phi, \vartheta)}{\partial \chi^{2}}+\frac{\partial^{2} \widetilde{I}_{020}(\chi, \eta, \phi, \vartheta)}{\partial \eta^{2}}+\frac{\partial^{2} \widetilde{I}_{020}(\chi, \eta, \phi, \vartheta)}{\partial \phi^{2}}\right]-
$$

$-\left[1+\varepsilon_{I, V} g_{I, V}(\chi, \eta, \phi, T)\right]\left[\widetilde{I}_{010}(\chi, \eta, \phi, \vartheta) \widetilde{V}_{000}(\chi, \eta, \phi, \vartheta)+\widetilde{I}_{000}(\chi, \eta, \phi, \vartheta) \widetilde{V}_{010}(\chi, \eta, \phi, \vartheta)\right]$ $\frac{\partial \widetilde{V}_{020}(\chi, \eta, \phi, \vartheta)}{\partial \vartheta}=\sqrt{\frac{D_{0 I}}{D_{0 V}}}\left[\frac{\partial^{2} \widetilde{V}_{020}(\chi, \eta, \phi, \vartheta)}{\partial \chi^{2}}+\frac{\partial^{2} \widetilde{V}_{020}(\chi, \eta, \phi, \vartheta)}{\partial \eta^{2}}+\frac{\partial^{2} \widetilde{V}_{020}(\chi, \eta, \phi, \vartheta)}{\partial \phi^{2}}\right]-$ $-\left[1+\varepsilon_{I, V} g_{I, V}(\chi, \eta, \phi, T)\right]\left[\widetilde{I}_{010}(\chi, \eta, \phi, \vartheta) \widetilde{V}_{000}(\chi, \eta, \phi, \vartheta)+\widetilde{I}_{000}(\chi, \eta, \phi, \vartheta) \widetilde{V}_{010}(\chi, \eta, \phi, \vartheta)\right] ;$ $\frac{\partial \widetilde{I}_{001}(\chi, \eta, \phi, \vartheta)}{\partial \vartheta}=\sqrt{\frac{D_{0 I}}{D_{0 V}}}\left[\frac{\partial^{2} \widetilde{I}_{001}(\chi, \eta, \phi, \vartheta)}{\partial \chi^{2}}+\frac{\partial^{2} \widetilde{I}_{001}(\chi, \eta, \phi, \vartheta)}{\partial \eta^{2}}+\frac{\partial^{2} \widetilde{I}_{001}(\chi, \eta, \phi, \vartheta)}{\partial \phi^{2}}\right]-$ $-\left[1+\varepsilon_{I, I} g_{I, I}(\chi, \eta, \phi, T)\right] \widetilde{I}_{000}^{2}(\chi, \eta, \phi, \vartheta)$

$\frac{\partial \widetilde{V}_{001}(\chi, \eta, \phi, \vartheta)}{\partial \vartheta}=\sqrt{\frac{D_{0 V}}{D_{0 I}}}\left[\frac{\partial^{2} \widetilde{V}_{001}(\chi, \eta, \phi, \vartheta)}{\partial \chi^{2}}+\frac{\partial^{2} \widetilde{V}_{001}(\chi, \eta, \phi, \vartheta)}{\partial \eta^{2}}+\frac{\partial^{2} \widetilde{V}_{001}(\chi, \eta, \phi, \vartheta)}{\partial \phi^{2}}\right]-$ $-\left[1+\varepsilon_{I, I} g_{I, I}(\chi, \eta, \phi, T)\right] \widetilde{V}_{000}^{2}(\chi, \eta, \phi, \vartheta) ;$ $\frac{\partial \widetilde{I}_{110}(\chi, \eta, \phi, \vartheta)}{\partial \vartheta}=\sqrt{\frac{D_{0 I}}{D_{0 V}}}\left[\frac{\partial^{2} \widetilde{I}_{110}(\chi, \eta, \phi, \vartheta)}{\partial \chi^{2}}+\frac{\partial^{2} \widetilde{I}_{110}(\chi, \eta, \phi, \vartheta)}{\partial \eta^{2}}+\frac{\partial^{2} \widetilde{I}_{110}(\chi, \eta, \phi, \vartheta)}{\partial \phi^{2}}\right]+\sqrt{\frac{D_{0 I}}{D_{0 V}}} \times$ $\times\left\{\frac{\partial}{\partial \chi}\left[g_{I}(\chi, \eta, \phi, T) \frac{\partial \widetilde{I}_{010}(\chi, \eta, \phi, \vartheta)}{\partial \chi}\right]+\frac{\partial}{\partial \eta}\left[g_{I}(\chi, \eta, \phi, T) \frac{\partial \widetilde{I}_{010}(\chi, \eta, \phi, \vartheta)}{\partial \eta}\right]+\right.$ 


$$
\begin{aligned}
& \left.+\frac{\partial}{\partial \phi}\left[g_{I}(\chi, \eta, \phi, T) \frac{\partial \widetilde{I}_{010}(\chi, \eta, \phi, \vartheta)}{\partial \phi}\right]\right\}-\left[1+\varepsilon_{I, I} g_{I, I}(\chi, \eta, \phi, T)\right] \times \\
& \times\left[\widetilde{I}_{100}(\chi, \eta, \phi, \vartheta) \widetilde{V}_{000}(\chi, \eta, \phi, \vartheta)+\widetilde{I}_{000}(\chi, \eta, \phi, \vartheta) \widetilde{V}_{100}(\chi, \eta, \phi, \vartheta)\right] \\
& \frac{\partial \widetilde{V}_{110}(\chi, \eta, \phi, \vartheta)}{\partial \vartheta}=\sqrt{\frac{D_{0 V}}{D_{0 I}}}\left[\frac{\partial^{2} \widetilde{V}_{110}(\chi, \eta, \phi, \vartheta)}{\partial \chi^{2}}+\frac{\partial^{2} \widetilde{V}_{110}(\chi, \eta, \phi, \vartheta)}{\partial \eta^{2}}+\frac{\partial^{2} \widetilde{V}_{110}(\chi, \eta, \phi, \vartheta)}{\partial \phi^{2}}\right]+ \\
& +\sqrt{\frac{D_{0 V}}{D_{0 I}}}\left\{\frac{\partial}{\partial \chi}\left[g_{V}(\chi, \eta, \phi, T) \frac{\partial \widetilde{V}_{010}(\chi, \eta, \phi, \vartheta)}{\partial \chi}\right]+\frac{\partial}{\partial \eta}\left[g_{V}(\chi, \eta, \phi, T) \frac{\partial \widetilde{V}_{010}(\chi, \eta, \phi, \vartheta)}{\partial \eta}\right]+\right. \\
& \left.+\frac{\partial}{\partial \phi}\left[g_{V}(\chi, \eta, \phi, T) \frac{\partial \widetilde{V}_{010}(\chi, \eta, \phi, \vartheta)}{\partial \phi}\right]\right\}-\left[1+\varepsilon_{V, V} g_{V, V}(\chi, \eta, \phi, T)\right] \times \\
& \times\left[\widetilde{V}_{100}(\chi, \eta, \phi, \vartheta) \widetilde{I}_{000}(\chi, \eta, \phi, \vartheta)+\widetilde{V}_{000}(\chi, \eta, \phi, \vartheta) \widetilde{I}_{100}(\chi, \eta, \phi, \vartheta)\right] ; \\
& \frac{\partial \widetilde{I}_{002}(\chi, \eta, \phi, \vartheta)}{\partial \vartheta}=\sqrt{\frac{D_{0 I}}{D_{0 V}}}\left[\frac{\partial^{2} \widetilde{I}_{002}(\chi, \eta, \phi, \vartheta)}{\partial \chi^{2}}+\frac{\partial^{2} \widetilde{I}_{002}(\chi, \eta, \phi, \vartheta)}{\partial \eta^{2}}+\frac{\partial^{2} \widetilde{I}_{002}(\chi, \eta, \phi, \vartheta)}{\partial \phi^{2}}\right]- \\
& -\left[1+\varepsilon_{I, I} g_{I, I}(\chi, \eta, \phi, T)\right] \widetilde{I}_{001}(\chi, \eta, \phi, \vartheta) \widetilde{I}_{000}(\chi, \eta, \phi, \vartheta) \\
& \frac{\partial \widetilde{V}_{002}(\chi, \eta, \phi, \vartheta)}{\partial \vartheta}=\sqrt{\frac{D_{0 V}}{D_{0 I}}}\left[\frac{\partial^{2} \widetilde{V}_{002}(\chi, \eta, \phi, \vartheta)}{\partial \chi^{2}}+\frac{\partial^{2} \widetilde{V}_{002}(\chi, \eta, \phi, \vartheta)}{\partial \eta^{2}}+\frac{\partial^{2} \widetilde{V}_{002}(\chi, \eta, \phi, \vartheta)}{\partial \phi^{2}}\right]- \\
& -\left[1+\varepsilon_{V, V} g_{V, V}(\chi, \eta, \phi, E)\right] \widetilde{V}_{001}(\chi, \eta, \phi, \vartheta) \widetilde{V}_{000}(\chi, \eta, \phi, \vartheta) ; \\
& \frac{\partial \widetilde{I}_{101}(\chi, \eta, \phi, \vartheta)}{\partial \vartheta}=\sqrt{\frac{D_{0 I}}{D_{0 V}}}\left[\frac{\partial^{2} \widetilde{I}_{101}(\chi, \eta, \phi, \vartheta)}{\partial \chi^{2}}+\frac{\partial^{2} \widetilde{I}_{101}(\chi, \eta, \phi, \vartheta)}{\partial \eta^{2}}+\frac{\partial^{2} \widetilde{I}_{101}(\chi, \eta, \phi, \vartheta)}{\partial \phi^{2}}\right]+ \\
& +\sqrt{\frac{D_{0 I}}{D_{0 V}}}\left\{\frac{\partial}{\partial \chi}\left[g_{I}(\chi, \eta, \phi, T) \frac{\partial \widetilde{I}_{001}(\chi, \eta, \phi, \vartheta)}{\partial \chi}\right]+\frac{\partial}{\partial \eta}\left[g_{I}(\chi, \eta, \phi, T) \frac{\partial \widetilde{I}_{001}(\chi, \eta, \phi, \vartheta)}{\partial \eta}\right]+\right. \\
& \left.+\frac{\partial}{\partial \phi}\left[g_{I}(\chi, \eta, \phi, T) \frac{\partial \widetilde{I}_{001}(\chi, \eta, \phi, \vartheta)}{\partial \phi}\right]\right\}-\left[1+\varepsilon_{I} g_{I}(\chi, \eta, \phi, T)\right] \widetilde{I}_{100}(\chi, \eta, \phi, \vartheta) \widetilde{V}_{000}(\chi, \eta, \phi, \vartheta) \\
& \frac{\partial \widetilde{V}_{101}(\chi, \eta, \phi, \vartheta)}{\partial \vartheta}=\sqrt{\frac{D_{0 V}}{D_{0 I}}}\left[\frac{\partial^{2} \widetilde{V}_{101}(\chi, \eta, \phi, \vartheta)}{\partial \chi^{2}}+\frac{\partial^{2} \widetilde{V}_{101}(\chi, \eta, \phi, \vartheta)}{\partial \eta^{2}}+\frac{\partial^{2} \widetilde{V}_{101}(\chi, \eta, \phi, \vartheta)}{\partial \phi^{2}}\right]+ \\
& +\sqrt{\frac{D_{0 V}}{D_{0 I}}}\left\{\frac{\partial}{\partial \chi}\left[g_{V}(\chi, \eta, \phi, T) \frac{\partial \widetilde{V}_{001}(\chi, \eta, \phi, \vartheta)}{\partial \chi}\right]+\frac{\partial}{\partial \eta}\left[g_{V}(\chi, \eta, \phi, T) \frac{\partial \widetilde{V}_{001}(\chi, \eta, \phi, \vartheta)}{\partial \eta}\right]+\right.
\end{aligned}
$$




$$
\begin{gathered}
\left.+\frac{\partial}{\partial \phi}\left[g_{V}(\chi, \eta, \phi, T) \frac{\partial \widetilde{V}_{001}(\chi, \eta, \phi, \vartheta)}{\partial \phi}\right]\right\}-\left[1+\varepsilon_{V} g_{V}(\chi, \eta, \phi, T)\right] \widetilde{I}_{000}(\chi, \eta, \phi, \vartheta) \widetilde{V}_{100}(\chi, \eta, \phi, \vartheta) ; \\
\frac{\partial \widetilde{I}_{011}(\chi, \eta, \phi, \vartheta)}{\partial \vartheta}=\sqrt{\frac{D_{0 I}}{D_{0 V}}}\left[\frac{\partial^{2} \widetilde{I}_{011}(\chi, \eta, \phi, \vartheta)}{\partial \chi^{2}}+\frac{\partial^{2} \widetilde{I}_{011}(\chi, \eta, \phi, \vartheta)}{\partial \eta^{2}}+\frac{\partial^{2} \widetilde{I}_{011}(\chi, \eta, \phi, \vartheta)}{\partial \phi^{2}}\right]-\widetilde{I}_{010}(\chi, \eta, \phi, \vartheta) \times \\
\times\left[1+\varepsilon_{I, I} g_{I, I}(\chi, \eta, \phi, T)\right] \widetilde{I}_{000}(\chi, \eta, \phi, \vartheta)-\left[1+\varepsilon_{I, V} g_{I, V}(\chi, \eta, \phi, T)\right] \widetilde{I}_{001}(\chi, \eta, \phi, \vartheta) \widetilde{V}_{000}(\chi, \eta, \phi, \vartheta) \\
\frac{\partial \widetilde{V}_{011}(\chi, \eta, \phi, \vartheta)}{\partial \vartheta}=\sqrt{\frac{D_{0 V}}{D_{0 I}}}\left[\frac{\partial^{2} \widetilde{V}_{011}(\chi, \eta, \phi, \vartheta)}{\partial \chi^{2}}+\frac{\partial^{2} \widetilde{V}_{011}(\chi, \eta, \phi, \vartheta)}{\partial \eta^{2}}+\frac{\partial^{2} \widetilde{V}_{011}(\chi, \eta, \phi, \vartheta)}{\partial \phi^{2}}\right]-\widetilde{V}_{010}(\chi, \eta, \phi, \vartheta) \times \\
\times\left[1+\varepsilon_{V, V} g_{V, V}(\chi, \eta, \phi, T)\right] \widetilde{V}_{000}(\chi, \eta, \phi, \vartheta)-\left[1+\varepsilon_{I, V} g_{I, V}(\chi, \eta, \phi, t)\right] \widetilde{I}_{000}(\chi, \eta, \phi, \vartheta) \widetilde{V}_{001}(\chi, \eta, \phi, \vartheta) ; \\
\left.\frac{\partial \widetilde{\rho}_{i j k}(\chi, \eta, \phi, \vartheta)}{\partial \chi}\right|_{x=0}=0,\left.\frac{\partial \widetilde{\rho}_{i j k}(\chi, \eta, \phi, \vartheta)}{\partial \chi}\right|_{x=1}=0,\left.\frac{\partial \widetilde{\rho}_{i j k}(\chi, \eta, \phi, \vartheta)}{\partial \eta}\right|_{\eta=0}=0, \\
\left.\frac{\partial \widetilde{\rho}_{i j k}(\chi, \eta, \phi, \vartheta)}{\partial \eta}\right|_{\eta=1}=0, \\
\left.\frac{\partial \widetilde{\rho}_{i j k}(\chi, \eta, \phi, \vartheta)}{\partial \phi}\right|_{\phi=0}=0,\left.\frac{\partial \widetilde{\rho}_{i j k}(\chi, \eta, \phi, \vartheta)}{\partial \phi}\right|_{\phi=1}=0 \quad(\mathrm{i} \geq 0, \mathrm{j} \geq 0, \mathrm{k} \geq 0) ; \\
\widetilde{\rho}_{000}(\chi, \eta, \phi, 0)=f_{\rho}(\chi, \eta, \phi) / \rho^{*}, \quad \widetilde{\rho}_{i j k}(\chi, \eta, \phi, 0)=0 \quad(\mathrm{i} \geq 1, \mathrm{j} \geq 1, \mathrm{k} \geq 1) .
\end{gathered}
$$

Solutions of the above equations could be written as

$$
\widetilde{\rho}_{000}(\chi, \eta, \phi, \vartheta)=\frac{1}{L}+\frac{2}{L} \sum_{n=1}^{\infty} F_{n \rho} c(\chi) c(\eta) c(\phi) e_{n \rho}(\vartheta),
$$

where $F_{n \rho}=\frac{1}{\rho^{*}} \int_{0}^{1} \cos (\pi n u) \int_{0}^{1} \cos (\pi n v) \int_{0}^{1} \cos (\pi n w) f_{n \rho}(u, v, w) d w d v d u, c_{n}(\chi)=\cos (\pi n \chi)$,

$$
e_{n I}(\vartheta)=\exp \left(-\pi^{2} n^{2} \vartheta \sqrt{D_{0 V} / D_{0 I}}\right), e_{n V}(\vartheta)=\exp \left(-\pi^{2} n^{2} \vartheta \sqrt{D_{0 I} / D_{0 V}}\right)
$$

$\widetilde{I}_{i 00}(\chi, \eta, \phi, \vartheta)=-2 \pi \sqrt{\frac{D_{0 I}}{D_{0 V}}} \sum_{n=1}^{\infty} n c_{n}(\chi) c(\eta) c(\phi) e_{n I}(\vartheta) \int_{0}^{\vartheta} e_{n I}(-\tau) \int_{0}^{1} s_{n}(u) \int_{0}^{1} c_{n}(v) \int_{0}^{1} \frac{\partial \widetilde{I}_{i-100}(u, v, w, \tau)}{\partial u} \times$ $\times c_{n}(w) g_{I}(u, v, w, T) d w d v d u d \tau-2 \pi \sqrt{\frac{D_{0 I}}{D_{0 V}}} \sum_{n=1}^{\infty} n c_{n}(\chi) c(\eta) c(\phi) e_{n I}(\vartheta) \int_{0}^{\vartheta} e_{n I}(-\tau) \int_{0}^{1} c_{n}(u) \int_{0}^{1} s_{n}(v) \times$ $\times \int_{0}^{1} c_{n}(w) g_{I}(u, v, w, T) \frac{\partial \widetilde{I}_{i-100}(u, v, w, \tau)}{\partial v} d w d v d u d \tau-2 \pi \sqrt{\frac{D_{0 I}}{D_{0 V}}} \sum_{n=1}^{\infty} n c_{n}(\chi) c(\eta) c(\phi) e_{n I}(\vartheta) \int_{0}^{\vartheta} e_{n I}(-\tau) \times$

$$
\times \int_{0}^{1} c_{n}(u) \int_{0}^{1} c_{n}(v) \int_{0}^{1} s_{n}(w) g_{I}(u, v, w, T) \frac{\partial \widetilde{I}_{i-100}(u, v, w, \tau)}{\partial w} d w d v d u d \tau, \mathrm{i} \geq 1,
$$


$\widetilde{V}_{i 00}(\chi, \eta, \phi, \vartheta)=-2 \pi \sqrt{\frac{D_{0 V}}{D_{0 I}}} \sum_{n=1}^{\infty} n c_{n}(\chi) c(\eta) c(\phi) e_{n V}(\vartheta) \int_{0}^{g} e_{n I}(-\tau) \int_{0}^{1} s_{n}(u) \int_{0}^{1} c_{n}(v) \int_{0}^{1} g_{V}(u, v, w, T) \times$ $\times c_{n}(w) \frac{\partial \widetilde{V}_{i-100}(u, \tau)}{\partial u} d w d v d u d \tau-\sqrt{\frac{D_{0 V}}{D_{0 I}}} \sum_{n=1}^{\infty} n c_{n}(\chi) c(\eta) c(\phi) e_{n V}(\vartheta) \int_{0}^{g} e_{n I}(-\tau) \int_{0}^{1} c_{n}(u) \int_{0}^{1} s_{n}(v) \times$ $\times 2 \pi \int_{0}^{1} c_{n}(w) g_{V}(u, v, w, T) \frac{\partial \widetilde{V}_{i-100}(u, \tau)}{\partial v} d w d v d u d \tau-2 \pi \sqrt{\frac{D_{0 V}}{D_{01}}} \sum_{n=1}^{\infty} n c_{n}(\chi) c(\eta) c(\phi) e_{n V}(\vartheta) \times$ $\times \int_{0}^{g} e_{n I}(-\tau) \int_{0}^{1} c_{n}(u) \int_{0}^{1} c_{n}(v) \int_{0}^{1} s_{n}(w) g_{V}(u, v, w, T) \frac{\partial \widetilde{\partial}_{i-100}(u, \tau)}{\partial w} d w d v d u d \tau, \mathrm{i} \geq 1$, where $s_{n}(\chi)=\sin (\pi n \chi)$;

$\widetilde{\rho}_{010}(\chi, \eta, \phi, \vartheta)=-2 \sum_{n=1}^{\infty} c_{n}(\chi) c_{n}(\eta) c_{n}(\phi) e_{n \rho}(\vartheta) \int_{0}^{\vartheta} e_{n \rho}(-\tau) \int_{0}^{1} c_{n}(u) \int_{0}^{1} c_{n}(v) \int_{0}^{1} c_{n}(w) \times$ $\times\left[1+\varepsilon_{I, V} g_{I, V}(u, v, w, T)\right] \widetilde{I}_{000}(u, v, w, \tau) \widetilde{V}_{000}(u, v, w, \tau) d w d v d u d \tau ;$

$\widetilde{\rho}_{020}(\chi, \eta, \phi, \vartheta)=-2 \sqrt{\frac{D_{01}}{D_{0 \nu}}} \sum_{n=1}^{\infty} c_{n}(\chi) c_{n}(\eta) c_{n}(\phi) e_{n \rho}(\vartheta) \int_{0}^{g} e_{n \rho}(-\tau) \int_{0}^{1} c_{n}(u) \int_{0}^{1} c_{n}(v) \int_{0}^{1} c_{n}(w)\left[1+\varepsilon_{I, V} \times\right.$ $\left.\times g_{I, v}(u, v, w, T)\right]\left[\widetilde{I}_{010}(u, v, w, \tau) \widetilde{V}_{000}(u, v, w, \tau)+\widetilde{I}_{000}(u, v, w, \tau) \widetilde{V}_{010}(u, v, w, \tau)\right] d w d v d u d \tau ;$

$$
\widetilde{\rho}_{001}(\chi, \eta, \phi, \vartheta)=-2 \sum_{n=1}^{\infty} c_{n}(\chi) c_{n}(\eta) c_{n}(\phi) e_{n \rho}(\vartheta) \int_{0}^{\vartheta} e_{n \rho}(-\tau) \int_{0}^{1} c_{n}(u) \int_{0}^{1} c_{n}(v) \int_{0}^{1} c_{n}(w) \times
$$
$\times\left[1+\varepsilon_{\rho, \rho} g_{\rho, \rho}(u, v, w, T)\right] \widetilde{\rho}_{000}^{2}(u, v, w, \tau) d w d v d u d \tau ;$

$\widetilde{\rho}_{002}(\chi, \eta, \phi, \vartheta)=-2 \sum_{n=1}^{\infty} c_{n}(\chi) c_{n}(\eta) c_{n}(\phi) e_{n \rho}(\vartheta) \int_{0}^{g} e_{n \rho}(-\tau) \int_{0}^{1} c_{n}(u) \int_{0}^{1} c_{n}(v) \int_{0}^{1} c_{n}(w) \times$ $\times\left\lfloor 1+\varepsilon_{\rho, \rho} g_{\rho, \rho}(u, v, w, T)\right] \widetilde{\rho}_{001}(u, v, w, \tau) \widetilde{\rho}_{000}(u, v, w, \tau) d w d v d u d \tau ;$

$\widetilde{I}_{110}(\chi, \eta, \phi, \vartheta)=-2 \pi \sqrt{\frac{D_{0 I}}{D_{0 V}}} \sum_{n=1}^{\infty} n c_{n}(\chi) c_{n}(\eta) c_{n}(\phi) e_{n I}(\vartheta) \int_{0}^{g} e_{n I}(-\tau) \int_{0}^{1} s_{n}(u) \int_{0}^{1} c_{n}(v) \int_{0}^{1} c_{n}(u) \times$

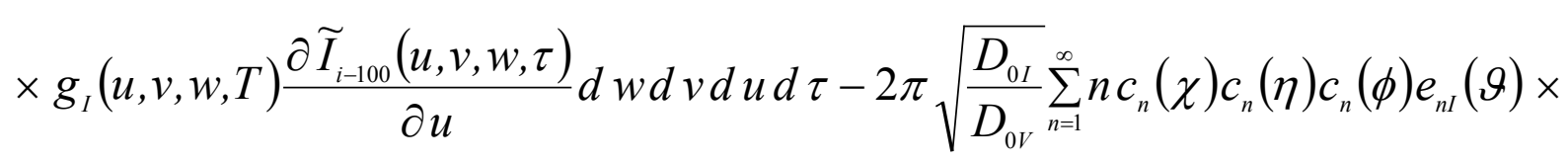
$\times \int_{0}^{g} e_{n I}(-\tau) \int_{0}^{1} c_{n}(u) \int_{0}^{1} s_{n}(v) \int_{0}^{1} c_{n}(u) g_{I}(u, v, w, T) \frac{\partial \widetilde{I}_{i-100}(u, v, w, \tau)}{\partial v} d w d v d u d \tau-2 \pi \sqrt{\frac{D_{0 I}}{D_{0 V}}} \times$ $\times \sum_{n=1}^{\infty} n e_{n I}(\vartheta) \int_{0}^{g} e_{n I}(-\tau) \int_{0}^{1} c_{n}(u) \int_{0}^{1} c_{n}(v) \int_{0}^{1} s_{n}(u) g_{I}(u, v, w, T) \frac{\partial \widetilde{I}_{i-100}(u, v, w, \tau)}{\partial w} d w d v d u d \tau \times$ $\times c_{n}(\chi) c_{n}(\eta) c_{n}(\phi)-2 \sum_{n=1}^{\infty} c_{n}(\chi) e_{n l}(\vartheta) c_{n}(\eta) c_{n}(\phi) \int_{0}^{g} e_{n I}(-\tau) \int_{0}^{1} c_{n}(u) \int_{0}^{1} c_{n}(v) \int_{0}^{1} c_{n}(v)\left[1+\varepsilon_{I, V} \times\right.$ 
$\left.\times g_{I, V}(u, v, w, T)\right]\left[\widetilde{I}_{100}(u, v, w, \tau) \widetilde{V}_{000}(u, v, w, \tau)+\widetilde{I}_{000}(u, v, w, \tau) \widetilde{V}_{100}(u, v, w, \tau)\right] d w d v d u d \tau$ $\widetilde{V}_{110}(\chi, \eta, \phi, \vartheta)=-2 \pi \sqrt{\frac{D_{0 V}}{D_{0 I}}} \sum_{n=1}^{\infty} n c_{n}(\chi) c_{n}(\eta) c_{n}(\phi) e_{n V}(\vartheta) \int_{0}^{\vartheta} e_{n V}(-\tau) \int_{0}^{1} s_{n}(u) \int_{0}^{1} c_{n}(v) \int_{0}^{1} c_{n}(u) \times$ $\times g_{V}(u, v, w, T) \frac{\partial \widetilde{V}_{i-100}(u, v, w, \tau)}{\partial u} d w d v d u d \tau-2 \pi \sqrt{\frac{D_{0 V}}{D_{0 I}}} \sum_{n=1}^{\infty} n c_{n}(\chi) c_{n}(\eta) c_{n}(\phi) e_{n V}(\vartheta) \times$ $\times \int_{0}^{g} e_{n V}(-\tau) \int_{0}^{1} c_{n}(u) \int_{0}^{1} s_{n}(v) \int_{0}^{1} c_{n}(u) g_{V}(u, v, w, T) \frac{\partial \widetilde{V}_{i-100}(u, v, w, \tau)}{\partial v} d w d v d u d \tau-2 \pi \sqrt{\frac{D_{0 V}}{D_{0 I}}} \times$ $\times \sum_{n=1}^{\infty} n e_{n V}(\vartheta) \int_{0}^{\vartheta} e_{n V}(-\tau) \int_{0}^{1} c_{n}(u) \int_{0}^{1} c_{n}(v) \int_{0}^{1} s_{n}(u) g_{V}(u, v, w, T) \frac{\partial \widetilde{V}_{i-100}(u, v, w, \tau)}{\partial w} d w d v d u d \tau \times$ $\times c_{n}(\chi) c_{n}(\eta) c_{n}(\phi)-2 \sum_{n=1}^{\infty} c_{n}(\chi) e_{n I}(\vartheta) c_{n}(\eta) c_{n}(\phi) \int_{0}^{9} e_{n V}(-\tau) \int_{0}^{1} c_{n}(u) \int_{0}^{1} c_{n}(v) \int_{0}^{1}\left[1+\varepsilon_{I, V} g_{I, V}(u, v, w, T)\right] \times$ $\times c_{n}(w)\left[\widetilde{I}_{100}(u, v, w, \tau) \widetilde{V}_{000}(u, v, w, \tau)+\widetilde{I}_{000}(u, v, w, \tau) \widetilde{V}_{100}(u, v, w, \tau)\right] d w d v d u d \tau ;$ $\widetilde{I}_{101}(\chi, \eta, \phi, \vartheta)=-2 \pi \sqrt{\frac{D_{0 I}}{D_{0 V}}} \sum_{n=1}^{\infty} n c_{n}(\chi) c_{n}(\eta) c_{n}(\phi) e_{n I}(\vartheta) \int_{0}^{\vartheta} e_{n I}(-\tau) \int_{0}^{1} s_{n}(u) \int_{0}^{1} c_{n}(v) \int_{0}^{1} g_{I}(u, v, w, T) \times$ $\times c_{n}(w) \frac{\partial \widetilde{I}_{001}(u, v, w, \tau)}{\partial u} d w d v d u d \tau-2 \pi \sqrt{\frac{D_{0 I}}{D_{0 V}}} \sum_{n=1}^{\infty} n c_{n}(\chi) c_{n}(\eta) c_{n}(\phi) e_{n I}(\vartheta) \times$ $\times \int_{0}^{1} s_{n}(v) \int_{0}^{1} c_{n}(w) g_{I}(u, v, w, T) \frac{\partial \widetilde{I}_{001}(u, v, w, \tau)}{\partial v} d w d v d u d \tau-2 \pi \sqrt{\frac{D_{0 I}}{D_{0 V}}} \sum_{n=1}^{\infty} n e_{n I}(\vartheta) c_{n}(\chi) c_{n}(\eta) c_{n}(\phi) \times$ $\times \int_{0}^{\vartheta} e_{n I}(-\tau) \int_{0}^{1} c_{n}(u) \int_{0}^{1} c_{n}(v) \int_{0}^{1} s_{n}(w) g_{I}(u, v, w, T) \frac{\partial \widetilde{I}_{001}(u, v, w, \tau)}{\partial w} d w d v d u d \tau-2 \sum_{n=1}^{\infty} c_{n}(\chi) c_{n}(\eta) c_{n}(\phi) \times$ $\times e_{n I}(\vartheta) \int_{0}^{g} e_{n I}(-\tau) \int_{0}^{1} c_{n}(u) \int_{0}^{1} c_{n}(v) \int_{0}^{1} c_{n}(w)\left[1+\varepsilon_{I, V} g_{I, V}(u, v, w, T)\right] \widetilde{I}_{100}(u, v, w, \tau) \widetilde{V}_{000}(u, v, w, \tau) d w d v d u d \tau$ $\widetilde{V}_{101}(\chi, \eta, \phi, \vartheta)=-2 \pi \sqrt{\frac{D_{0 V}}{D_{0 I}}} \sum_{n=1}^{\infty} n c_{n}(\chi) c_{n}(\eta) c_{n}(\phi) e_{n V}(\vartheta) \int_{0}^{\vartheta} e_{n V}(-\tau) \int_{0}^{1} s_{n}(u) \int_{0}^{1} c_{n}(v) \int_{0}^{1} g_{V}(u, v, w, T) \times$ $\times c_{n}(w) \frac{\partial \widetilde{V}_{001}(u, v, w, \tau)}{\partial u} d w d v d u d \tau-2 \pi \sqrt{\frac{D_{0 V}}{D_{0 I}}} \sum_{n=1}^{\infty} n c_{n}(\chi) c_{n}(\eta) c_{n}(\phi) e_{n I}(\vartheta) \int_{0}^{\vartheta} e_{n V}(-\tau) \int_{0}^{1} c_{n}(u) \times$ $\times \int_{0}^{1} s_{n}(v) \int_{0}^{1} c_{n}(w) g_{I}(u, v, w, T) \frac{\partial \widetilde{I}_{001}(u, v, w, \tau)}{\partial v} d w d v d u d \tau-2 \pi \sqrt{\frac{D_{0 I}}{D_{0 V}}} \sum_{n=1}^{\infty} n e_{n I}(\vartheta) c_{n}(\chi) c_{n}(\eta) c_{n}(\phi) \times$ $\times \int_{0}^{\vartheta} e_{n V}(-\tau) \int_{0}^{1} c_{n}(u) \int_{0}^{1} c_{n}(v) \int_{0}^{1} s_{n}(w) g_{V}(u, v, w, T) \frac{\partial \widetilde{V}_{001}(u, v, w, \tau)}{\partial w} d w d v d u d \tau-2 \sum_{n=1}^{\infty} c_{n}(\chi) c_{n}(\eta) c_{n}(\phi) \times$ 
$\times e_{n V}(\vartheta) \int_{0}^{g} e_{n V}(-\tau) \int_{0}^{1} c_{n}(u) \int_{0}^{1} c_{n}(v) \int_{0}^{1} c_{n}(w)\left[1+\varepsilon_{I, V} g_{I, V}(u, v, w, T)\right] \widetilde{I}_{100}(u, v, w, \tau) \widetilde{V}_{000}(u, v, w, \tau) d w d v d u d \tau ;$ $\widetilde{I}_{011}(\chi, \eta, \phi, \vartheta)=-2 \sum_{n=1}^{\infty} c_{n}(\chi) c_{n}(\eta) c_{n}(\phi) e_{n I}(\vartheta) \int_{0}^{\vartheta} e_{n I}(-\tau) \int_{0}^{1} c_{n}(u) \int_{0}^{1} c_{n}(v) \int_{0}^{1} c_{n}(w)\left\{\widetilde{I}_{000}(u, v, w, \tau) \times\right.$ $\left.\times\left[1+\varepsilon_{I, I} g_{I, I}(u, v, w, T)\right] \tilde{I}_{010}(u, v, w, \tau)+\left[1+\varepsilon_{I, V} g_{I, V}(u, v, w, T)\right] \widetilde{I}_{001}(u, v, w, \tau) \widetilde{V}_{000}(u, v, w, \tau)\right\} d w d v d u d \tau$ $\widetilde{V}_{011}(\chi, \eta, \phi, \vartheta)=-2 \sum_{n=1}^{\infty} c_{n}(\chi) c_{n}(\eta) c_{n}(\phi) e_{n V}(\vartheta) \int_{0}^{\vartheta} e_{n V}(-\tau) \int_{0}^{1} c_{n}(u) \int_{0}^{1} c_{n}(v) \int_{0}^{1} c_{n}(w)\left\{\widetilde{I}_{000}(u, v, w, \tau) \times\right.$ $\left.\times\left[1+\varepsilon_{I, I} g_{I, I}(u, v, w, T)\right] \widetilde{I}_{010}(u, v, w, \tau)+\left[1+\varepsilon_{I, V} g_{I, V}(u, v, w, T)\right] \widetilde{I}_{001}(u, v, w, \tau) \widetilde{V}_{000}(u, v, w, \tau)\right\} d w d v d u d \tau$. Equations for functions $\Phi_{\rho i}(x, y, z, t), i \geq 0$ to describe concentrations of simplest complexes of radiation defects.

$$
\begin{aligned}
& \frac{\partial \Phi_{I 0}(x, y, z, t)}{\partial t}=D_{0 \Phi I}\left[\frac{\partial^{2} \Phi_{I 0}(x, y, z, t)}{\partial x^{2}}+\frac{\partial^{2} \Phi_{I 0}(x, y, z, t)}{\partial y^{2}}+\frac{\partial^{2} \Phi_{I 0}(x, y, z, t)}{\partial z^{2}}\right]+ \\
& +k_{I, I}(x, y, z, T) I^{2}(x, y, z, t)-k_{I}(x, y, z, T) I(x, y, z, t) \\
& \frac{\partial \Phi_{V 0}(x, y, z, t)}{\partial t}=D_{0 \Phi V}\left[\frac{\partial^{2} \Phi_{V 0}(x, y, z, t)}{\partial x^{2}}+\frac{\partial^{2} \Phi_{V 0}(x, y, z, t)}{\partial y^{2}}+\frac{\partial^{2} \Phi_{V 0}(x, y, z, t)}{\partial z^{2}}\right]+ \\
& +k_{V, V}(x, y, z, T) V^{2}(x, y, z, t)-k_{V}(x, y, z, T) V(x, y, z, t) ; \\
& \frac{\partial \Phi_{I i}(x, y, z, t)}{\partial t}=D_{0 \Phi I}\left[\frac{\partial^{2} \Phi_{I i}(x, y, z, t)}{\partial x^{2}}+\frac{\partial^{2} \Phi_{I i}(x, y, z, t)}{\partial y^{2}}+\frac{\partial^{2} \Phi_{I i}(x, y, z, t)}{\partial z^{2}}\right]+ \\
& +D_{0 \Phi I}\left\{\frac{\partial}{\partial x}\left[g_{\Phi I}(x, y, z, T) \frac{\partial \Phi_{I i-1}(x, y, z, t)}{\partial x}\right]+\frac{\partial}{\partial y}\left[g_{\Phi I}(x, y, z, T) \frac{\partial \Phi_{I i-1}(x, y, z, t)}{\partial y}\right]+\right. \\
& \left.+\frac{\partial}{\partial z}\left[g_{\Phi I}(x, y, z, T) \frac{\partial \Phi_{I i-1}(x, y, z, t)}{\partial z}\right]\right\}, \mathrm{i} \geq 1, \\
& \frac{\partial \Phi_{V i}(x, y, z, t)}{\partial t}=D_{0 \Phi V}\left[\frac{\partial^{2} \Phi_{V i}(x, y, z, t)}{\partial x^{2}}+\frac{\partial^{2} \Phi_{V i}(x, y, z, t)}{\partial y^{2}}+\frac{\partial^{2} \Phi_{V i}(x, y, z, t)}{\partial z^{2}}\right]+ \\
& +D_{0 \Phi V}\left\{\frac{\partial}{\partial x}\left[g_{\Phi V}(x, y, z, T) \frac{\partial \Phi_{V i-1}(x, y, z, t)}{\partial x}\right]+\frac{\partial}{\partial y}\left[g_{\Phi V}(x, y, z, T) \frac{\partial \Phi_{V i-1}(x, y, z, t)}{\partial y}\right]+\right. \\
& \left.+\frac{\partial}{\partial z}\left[g_{\Phi V}(x, y, z, T) \frac{\partial \Phi_{V i-1}(x, y, z, t)}{\partial z}\right]\right\}, \mathrm{i} \geq 1 ;
\end{aligned}
$$

Boundary and initial conditions for the functions takes the form

$\left.\frac{\partial \Phi_{\rho i}(x, y, z, t)}{\partial x}\right|_{x=0}=0,\left.\frac{\partial \Phi_{\rho i}(x, y, z, t)}{\partial x}\right|_{x=L_{x}}=0,\left.\frac{\partial \Phi_{\rho i}(x, y, z, t)}{\partial y}\right|_{y=0}=0,\left.\frac{\partial \Phi_{\rho i}(x, y, z, t)}{\partial y}\right|_{y=L_{y}}=0$, 


$$
\left.\frac{\partial \Phi_{\rho i}(x, y, z, t)}{\partial z}\right|_{z=0}=0,\left.\frac{\partial \Phi_{\rho i}(x, y, z, t)}{\partial z}\right|_{z=L_{z}}=0, \mathrm{i} \geq 0 ; \Phi_{\rho 0}(\mathrm{x}, \mathrm{y}, \mathrm{z}, 0)=\mathrm{f}_{\Phi_{\rho}}(\mathrm{x}, \mathrm{y}, \mathrm{z}),
$$

Solutions of the above equations could be written as

$$
\begin{gathered}
\Phi_{\rho 0}(x, y, z, t)=\frac{1}{L_{x} L_{y} L_{z}}+\frac{2}{L_{x} L_{y} L_{z}} \sum_{n=1}^{\infty} F_{n \Phi_{\rho}} c_{n}(x) c_{n}(y) c_{n}(z) e_{n \Phi_{\rho}}(t)+\frac{2}{L} \sum_{n=1}^{\infty} n c_{n}(x) c_{n}(y) c_{n}(z) \times \\
\times e_{\Phi_{\rho^{n}}}(t) \int_{0}^{t} e_{\Phi_{\rho^{n}}}(-\tau) \int_{0}^{L_{x}} c_{n}(u) \int_{0}^{L_{y}} c_{n}(v) \int_{0}^{L_{z}} c_{n}(w)\left[k_{I, I}(u, v, w, T) I^{2}(u, v, w, \tau)-\right. \\
\left.-k_{I}(u, v, w, T) I(u, v, w, \tau)\right] d w d v d u d \tau,
\end{gathered}
$$

where $F_{n \Phi_{\rho}}=\int_{0}^{L_{x}} c_{n}(u) \int_{0}^{L_{y}} c_{n}(v) \int_{0}^{L_{z}} c_{n}(w) f_{\Phi_{\rho}}(u, v, w) d w d v d u, e_{n \Phi_{\rho}}(t)=\exp \left[-\pi^{2} n^{2} D_{0 \Phi_{\rho}} t\left(L_{x}^{-2}+L_{y}^{-2}+L_{z}^{-2}\right)\right], c_{n}(x)=\cos (\pi n$ $\left.x / L_{x}\right)$

$\Phi_{\rho i}(x, y, z, t)=-\frac{2 \pi}{L_{x}^{2} L_{y} L_{z}} \sum_{n=1}^{\infty} n c_{n}(x) c_{n}(y) c_{n}(z) e_{\Phi_{\rho^{n}}}(t) \int_{0}^{t} e_{\Phi_{\rho^{n}}}(-\tau) \int_{0}^{L_{x}} s_{n}(u) \int_{0}^{L_{y}} c_{n}(v) \int_{0}^{L_{z}} g_{\Phi_{\rho}}(u, v, w, T) \times$ $\times c_{n}(w) \frac{\partial \Phi_{I_{\rho} i-1}(u, v, w, \tau)}{\partial u} d w d v d u d \tau-\frac{2 \pi}{L_{x} L_{y}^{2} L_{z}} \sum_{n=1}^{\infty} n c_{n}(x) c_{n}(y) c_{n}(z) e_{\Phi_{\rho^{n}}}(t) \int_{0}^{t} e_{\Phi_{\rho^{n}}}(-\tau) \times$ $\times \int_{0}^{t} e_{\Phi_{\rho^{n}}}(-\tau) \int_{0}^{L_{x}} c_{n}(u) \int_{0}^{L_{y}} s_{n}(v) \int_{0}^{L_{z}} c_{n}(w) g_{\Phi_{\rho}}(u, v, w, T) \frac{\partial \Phi_{I_{\rho} i-1}(u, v, w, \tau)}{\partial v} d w d v d u d \tau-\frac{2 \pi}{L_{x} L_{y} L_{z}^{2}} \sum_{n=1}^{\infty} n \times$ $\times e_{\Phi_{\rho^{n}}}(t) \int_{0}^{t} e_{\Phi_{\rho^{n}}}(-\tau) \int_{0}^{L_{x}} c_{n}(u) \int_{0}^{L_{y}} c_{n}(v) \int_{0}^{L_{z}} s_{n}(w) \frac{\partial \Phi_{I_{\rho} i-1}(u, v, w, \tau)}{\partial w} g_{\Phi_{\rho}}(u, v, w, T) d w d v d u d \tau \times$

$$
\begin{gathered}
\times c_{n}(x) c_{n}(y) c_{n}(z), \mathrm{i} \geq 1, \\
\text { where } s_{n}(x)=\sin \left(\pi n x / L_{x}\right) .
\end{gathered}
$$

Equations for the functions $C_{i j}(x, y, z, t)(i \geq 0, j \geq 0)$, boundary and initial conditions could be written as

$$
\begin{gathered}
\frac{\partial C_{00}(x, y, z, t)}{\partial t}=D_{0 L} \frac{\partial^{2} C_{00}(x, y, z, t)}{\partial x^{2}}+D_{0 L} \frac{\partial^{2} C_{00}(x, y, z, t)}{\partial y^{2}}+D_{0 L} \frac{\partial^{2} C_{00}(x, y, z, t)}{\partial z^{2}} \\
\frac{\partial C_{i 0}(x, y, z, t)}{\partial t}=D_{0 L}\left[\frac{\partial^{2} C_{i 0}(x, y, z, t)}{\partial x^{2}}+\frac{\partial^{2} C_{i 0}(x, y, z, t)}{\partial y^{2}}+\frac{\partial^{2} C_{i 0}(x, y, z, t)}{\partial z^{2}}\right]+ \\
+D_{0 L} \frac{\partial}{\partial x}\left[g_{L}(x, y, z, T) \frac{\partial C_{i-10}(x, y, z, t)}{\partial x}\right]+D_{0 L} \frac{\partial}{\partial y}\left[g_{L}(x, y, z, T) \frac{\partial C_{i-10}(x, y, z, t)}{\partial y}\right]+ \\
+D_{0 L} \frac{\partial}{\partial z}\left[g_{L}(x, y, z, T) \frac{\partial C_{i-10}(x, y, z, t)}{\partial z}\right], \mathrm{i} \geq 1
\end{gathered}
$$




$$
\begin{aligned}
& \frac{\partial C_{01}(x, y, z, t)}{\partial t}=D_{0 L} \frac{\partial^{2} C_{01}(x, y, z, t)}{\partial x^{2}}+D_{0 L} \frac{\partial^{2} C_{01}(x, y, z, t)}{\partial y^{2}}+D_{0 L} \frac{\partial^{2} C_{01}(x, y, z, t)}{\partial z^{2}}+ \\
& +D_{0 L} \frac{\partial}{\partial x}\left[\frac{C_{00}^{\gamma}(x, y, z, t)}{P^{\gamma}(x, y, z, T)} \frac{\partial C_{00}(x, y, z, t)}{\partial x}\right]+D_{0 L} \frac{\partial}{\partial y}\left[\frac{C_{00}^{\gamma}(x, y, z, t)}{P^{\gamma}(x, y, z, T)} \frac{\partial C_{00}(x, y, z, t)}{\partial y}\right]+ \\
& +D_{0 L} \frac{\partial}{\partial z}\left[\frac{C_{00}^{\gamma}(x, y, z, t)}{P^{\gamma}(x, y, z, T)} \frac{\partial C_{00}(x, y, z, t)}{\partial z}\right] ; \\
& \frac{\partial C_{02}(x, y, z, t)}{\partial t}=D_{0 L} \frac{\partial^{2} C_{02}(x, y, z, t)}{\partial x^{2}}+D_{0 L} \frac{\partial^{2} C_{02}(x, y, z, t)}{\partial y^{2}}+D_{0 L} \frac{\partial^{2} C_{02}(x, y, z, t)}{\partial z^{2}}+ \\
& +D_{0 L}\left\{\frac{\partial}{\partial x}\left[C_{01}(x, y, z, t) \frac{C_{00}^{\gamma-1}(x, y, z, t)}{P^{\gamma}(x, y, z, T)} \frac{\partial C_{00}(x, y, z, t)}{\partial x}\right]+\frac{\partial}{\partial y}\left[C_{01}(x, y, z, t) \frac{C_{00}^{\gamma-1}(x, y, z, t)}{P^{\gamma}(x, y, z, T)} \times\right.\right. \\
& \left.\left.\times \frac{\partial C_{00}(x, y, z, t)}{\partial y}\right]+\frac{\partial}{\partial z}\left[C_{01}(x, y, z, t) \frac{C_{00}^{\gamma-1}(x, y, z, t)}{P^{\gamma}(x, y, z, T)} \frac{\partial C_{00}(x, y, z, t)}{\partial z}\right]\right\}+ \\
& \left.\left.\times \frac{\partial C_{00}(x, y, z, t)}{\partial y}\right]+\frac{\partial}{\partial z}\left[C_{01}(x, y, z, t) \frac{C_{00}^{\gamma-1}(x, y, z, t)}{P^{\gamma}(x, y, z, T)} \frac{\partial C_{00}(x, y, z, t)}{\partial z}\right]\right\}+D_{0 L}\left\{\frac { \partial } { \partial x } \left[\frac{C_{00}^{\gamma}(x, y, z, t)}{P^{\gamma}(x, y, z, T)} \times\right.\right. \\
& \left.\left.\times \frac{\partial C_{01}(x, y, z, t)}{\partial x}\right]+\frac{\partial}{\partial y}\left[\frac{C_{00}^{\gamma}(x, y, z, t)}{P^{\gamma}(x, y, z, T)} \frac{\partial C_{01}(x, y, z, t)}{\partial y}\right]+\frac{\partial}{\partial z}\left[\frac{C_{00}^{\gamma}(x, y, z, t)}{P^{\gamma}(x, y, z, T)} \frac{\partial C_{01}(x, y, z, t)}{\partial z}\right]\right\} ; \\
& \frac{\partial C_{11}(x, y, z, t)}{\partial t}=D_{0 L} \frac{\partial^{2} C_{11}(x, y, z, t)}{\partial x^{2}}+D_{0 L} \frac{\partial^{2} C_{11}(x, y, z, t)}{\partial y^{2}}+D_{0 L} \frac{\partial^{2} C_{11}(x, y, z, t)}{\partial z^{2}}+ \\
& +\left\{\frac{\partial}{\partial x}\left[C_{10}(x, y, z, t) \frac{C_{00}^{\gamma-1}(x, y, z, t)}{P^{\gamma}(x, y, z, T)} \frac{\partial C_{00}(x, y, z, t)}{\partial x}\right]+\frac{\partial}{\partial y}\left[C_{10}(x, y, z, t) \frac{C_{00}^{\gamma-1}(x, y, z, t)}{P^{\gamma}(x, y, z, T)} \times\right.\right. \\
& \left.\left.\times \frac{\partial C_{00}(x, y, z, t)}{\partial y}\right]+\frac{\partial}{\partial z}\left[C_{10}(x, y, z, t) \frac{C_{00}^{\gamma-1}(x, y, z, t)}{P^{\gamma}(x, y, z, T)} \frac{\partial C_{00}(x, y, z, t)}{\partial z}\right]\right\} D_{0 L}+ \\
& +D_{0 L}\left\{\frac{\partial}{\partial x}\left[\frac{C_{00}^{\gamma}(x, y, z, t)}{P^{\gamma}(x, y, z, T)} \frac{\partial C_{10}(x, y, z, t)}{\partial x}\right]+\frac{\partial}{\partial y}\left[\frac{C_{00}^{\gamma}(x, y, z, t)}{P^{\gamma}(x, y, z, T)} \frac{\partial C_{10}(x, y, z, t)}{\partial y}\right]+\right. \\
& \left.+\frac{\partial}{\partial z}\left[\frac{C_{00}^{\gamma}(x, y, z, t)}{P^{\gamma}(x, y, z, T)} \frac{\partial C_{10}(x, y, z, t)}{\partial z}\right]\right\}+D_{0 L}\left\{\frac{\partial}{\partial x}\left[g_{L}(x, y, z, T) \frac{\partial C_{01}(x, y, z, t)}{\partial x}\right]+\right. \\
& \left.+\frac{\partial}{\partial y}\left[g_{L}(x, y, z, T) \frac{\partial C_{01}(x, y, z, t)}{\partial y}\right]+\frac{\partial}{\partial z}\left[g_{L}(x, y, z, T) \frac{\partial C_{01}(x, y, z, t)}{\partial z}\right]\right\} ;
\end{aligned}
$$




$$
\begin{gathered}
\left.\frac{\partial C_{i j}(x, y, z, t)}{\partial x}\right|_{x=0}=0,\left.\frac{\partial C_{i j}(x, y, z, t)}{\partial x}\right|_{x=L_{x}}=0,\left.\frac{\partial C_{i j}(x, y, z, t)}{\partial y}\right|_{y=0}=0,\left.\frac{\partial C_{i j}(x, y, z, t)}{\partial y}\right|_{y=L_{y}}=0, \\
\left.\frac{\partial C_{i j}(x, y, z, t)}{\partial z}\right|_{z=0}=0,\left.\frac{\partial C_{i j}(x, y, z, t)}{\partial z}\right|_{z=L_{z}}=0, \mathrm{i} \geq 0, \mathrm{j} \geq 0 ; \\
\mathrm{C}_{00}(\mathrm{x}, \mathrm{y}, \mathrm{z}, 0)=\mathrm{f}_{\mathrm{C}}(\mathrm{x}, \mathrm{y}, \mathrm{z}), \mathrm{C}_{\mathrm{ij}}(\mathrm{x}, \mathrm{y}, \mathrm{z}, 0)=0, \mathrm{i} \geq 1, \mathrm{j} \geq 1 .
\end{gathered}
$$

Functions $C_{i j}(x, y, z, t)(i \geq 0, j \geq 0)$ could be approximated by the following series during solutions of the above equations

$$
C_{00}(x, y, z, t)=\frac{F_{0 C}}{L_{x} L_{y} L_{z}}+\frac{2}{L_{x} L_{y} L_{z}} \sum_{n=1}^{\infty} F_{n C} c_{n}(x) c_{n}(y) c_{n}(z) e_{n C}(t)
$$

Here

$$
e_{n C}(t)=\exp \left[-\pi^{2} n^{2} D_{0 c} t\left(\frac{1}{L_{x}^{2}}+\frac{1}{L_{y}^{2}}+\frac{1}{L_{z}^{2}}\right)\right], F_{n C}=\int_{0}^{L_{x}} c_{n}(u) \int_{0}^{L_{y}} c_{n}(v) \int_{0}^{L_{z}} f_{C}(u, v, w) c_{n}(w) d w d v d u ;
$$$$
C_{i 0}(x, y, z, t)=-\frac{2 \pi}{L_{x}^{2} L_{y} L_{z}} \sum_{n=1}^{\infty} n F_{n C} c_{n}(x) c_{n}(y) c_{n}(z) e_{n C}(t) \int_{0}^{t} e_{n C}(-\tau) \int_{0}^{L_{x}} s_{n}(u) \int_{0}^{L_{y}} c_{n}(v) \int_{0}^{L_{z}} g_{L}(u, v, w, T) \times
$$$$
\times c_{n}(w) \frac{\partial C_{i-10}(u, v, w, \tau)}{\partial u} d w d v d u d \tau-\frac{2 \pi}{L_{x} L_{y}^{2} L_{z}} \sum_{n=1}^{\infty} n F_{n C} c_{n}(x) c_{n}(y) c_{n}(z) e_{n C}(t) \int_{0}^{t} e_{n C}(-\tau) \times
$$

$\times \int_{0}^{L_{x}} c_{n}(u) \int_{0}^{L_{y}} S_{n}(v) \int_{0}^{L_{z}} c_{n}(v) g_{L}(u, v, w, T) \frac{\partial C_{i-10}(u, v, w, \tau)}{\partial v} d w d v d u d \tau-\frac{2 \pi}{L_{x} L_{y} L_{z}^{2}} \sum_{n=1}^{\infty} n F_{n C} e_{n C}(t) \times$ $\times c_{n}(x) c_{n}(y) c_{n}(z) \int_{0}^{t} e_{n C}(-\tau) \int_{0}^{L_{x}} c_{n}(u) \int_{0}^{L_{y}} c_{n}(v) \int_{0}^{L_{z}} s_{n}(v) g_{L}(u, v, w, T) \frac{\partial C_{i-10}(u, v, w, \tau)}{\partial w} d w d v d u d \tau, \mathrm{i} \geq 1 ;$ $C_{01}(x, y, z, t)=-\frac{2 \pi}{L_{x}^{2} L_{y} L_{z}} \sum_{n=1}^{\infty} n F_{n C} c_{n}(x) c_{n}(y) c_{n}(z) e_{n C}(t) \int_{0}^{t} e_{n C}(-\tau) \int_{0}^{L_{x}} S_{n}(u) \int_{0}^{L_{y}} c_{n}(v) \int_{0}^{L_{z}} c_{n}(w) \times$ $\times \frac{C_{00}^{\gamma}(u, v, w, \tau)}{P^{\gamma}(u, v, w, T)} \frac{\partial C_{00}(u, v, w, \tau)}{\partial u} d w d v d u d \tau-\frac{2 \pi}{L_{x} L_{y}^{2} L_{z}} \sum_{n=1}^{\infty} n F_{n C} c_{n}(x) c_{n}(y) c_{n}(z) e_{n C}(t) \times$ $\times \int_{0}^{t} e_{n C}(-\tau) \int_{0}^{L_{x}} c_{n}(u) \int_{0}^{L_{y}} S_{n}(v) \int_{0}^{L_{z}} c_{n}(w) \frac{C_{00}^{\gamma}(u, v, w, \tau)}{P^{\gamma}(u, v, w, T)} \frac{\partial C_{00}(u, v, w, \tau)}{\partial v} d w d v d u d \tau-\frac{2 \pi}{L_{x} L_{y} L_{z}^{2}} \sum_{n=1}^{\infty} n e_{n C}(t) \times$ $\times F_{n C} c_{n}(x) c_{n}(y) c_{n}(z) \int_{0}^{t} e_{n C}(-\tau) \int_{0}^{L_{x}} c_{n}(u) \int_{0}^{L_{y}} c_{n}(v) \int_{0}^{L_{z}} s_{n}(w) \frac{C_{00}^{\gamma}(u, v, w, \tau)}{P^{\gamma}(u, v, w, T)} \frac{\partial C_{00}(u, v, w, \tau)}{\partial w} d w d v d u d \tau ;$ $C_{02}(x, y, z, t)=-\frac{2 \pi}{L_{x}^{2} L_{y} L_{z}} \sum_{n=1}^{\infty} n F_{n C} c_{n}(x) c_{n}(y) c_{n}(z) e_{n C}(t) \int_{0}^{t} e_{n C}(-\tau) \int_{0}^{L_{x}} s_{n}(u) \int_{0}^{L_{y}} c_{n}(v) \int_{0}^{L_{z}} c_{n}(w) \times$ $\times C_{01}(u, v, w, \tau) \frac{C_{00}^{\gamma-1}(u, v, w, \tau)}{P^{\gamma}(u, v, w, T)} \frac{\partial C_{00}(u, v, w, \tau)}{\partial u} d w d v d u d \tau-\frac{2 \pi}{L_{x} L_{y}^{2} L_{z}} \sum_{n=1}^{\infty} F_{n C} c_{n}(x) c_{n}(y) \times$ 
$\times n c_{n}(z) e_{n C}(t) \int_{0}^{t} e_{n C}(-\tau) \int_{0}^{L_{x}} c_{n}(u) \int_{0}^{L_{y}} s_{n}(v) \int_{0}^{L_{z}} C_{01}(u, v, w, \tau) \frac{C_{00}^{\gamma-1}(u, v, w, \tau)}{P^{\gamma}(u, v, w, T)} \frac{\partial C_{00}(u, v, w, \tau)}{\partial v} \times$ $\times c_{n}(w) d w d v d u d \tau-\frac{2 \pi}{L_{x} L_{y} L_{z}^{2}} \sum_{n=1}^{\infty} n F_{n C} c_{n}(x) c_{n}(y) c_{n}(z) e_{n C}(t) \int_{0}^{t} e_{n C}(-\tau) \int_{0}^{L_{x}} c_{n}(u) \int_{0}^{L_{y}} c_{n}(v) \times$ $\times \int_{0}^{L_{z}} S_{n}(w) C_{01}(u, v, w, \tau) \frac{C_{00}^{\gamma-1}(u, v, w, \tau)}{P^{\gamma}(u, v, w, T)} \frac{\partial C_{00}(u, v, w, \tau)}{\partial w} d w d v d u d \tau-\frac{2 \pi}{L_{x}^{2} L_{y} L_{z}} \sum_{n=1}^{\infty} n c_{n}(x) \times$ $\times F_{n C} c_{n}(y) c_{n}(z) e_{n C}(t) \int_{0}^{t} e_{n C}(-\tau) \int_{0}^{L_{x}} S_{n}(u) \int_{0}^{L_{y}} c_{n}(v) \int_{0}^{L_{z}} c_{n}(w) C_{01}(u, v, w, \tau) \frac{\partial C_{00}(u, v, w, \tau)}{\partial u} \times$ $\times \frac{C_{00}^{\gamma-1}(u, v, w, \tau)}{P^{\gamma}(u, v, w, T)} d w d v d u d \tau-\frac{2 \pi}{L_{x} L_{y}^{2} L_{z}} \sum_{n=1}^{\infty} n F_{n C} c_{n}(x) c_{n}(y) c_{n}(z) e_{n C}(t) \int_{0}^{t} e_{n C}(-\tau) \int_{0}^{L_{x}} c_{n}(u) \times$ $\times \int_{0}^{L_{y}} S_{n}(v) \int_{0}^{L_{z}} c_{n}(w) C_{01}(u, v, w, \tau) \frac{C_{00}^{\gamma-1}(u, v, w, \tau)}{P^{\gamma}(u, v, w, T)} \frac{\partial C_{00}(u, v, w, \tau)}{\partial v} d w d v d u d \tau-\frac{2 \pi}{L_{x} L_{y} L_{z}^{2}} \sum_{n=1}^{\infty} n \times$ $\times F_{n C} c_{n}(x) c_{n}(y) c_{n}(z) e_{n C}(t) \int_{0}^{t} e_{n C}(-\tau) \int_{0}^{L_{x}} c_{n}(u) \int_{0}^{L_{y}} c_{n}(v) \int_{0}^{L_{z}} s_{n}(w) C_{01}(u, v, w, \tau) \frac{C_{00}^{\gamma-1}(u, v, w, \tau)}{P^{\gamma}(u, v, w, T)} \times$ $\times \frac{\partial C_{00}(u, v, w, \tau)}{\partial w} d w d v d u d \tau-\frac{2 \pi}{L_{x}^{2} L_{y} L_{z}} \sum_{n=1}^{\infty} F_{n C} c_{n}(x) c_{n}(y) c_{n}(z) e_{n C}(t) \int_{0}^{t} e_{n C}(-\tau) \int_{0}^{L_{x}} S_{n}(u) \times$ $\times n \int_{0}^{L_{y}} c_{n}(v) \int_{0}^{L_{z}} c_{n}(w) \frac{C_{00}^{\gamma}(u, v, w, \tau)}{P^{\gamma}(u, v, w, T)} \frac{\partial C_{01}(u, v, w, \tau)}{\partial u} d w d v d u d \tau-\frac{2 \pi}{L_{x} L_{y}^{2} L_{z}} \sum_{n=1}^{\infty} c_{n}(x) e_{n C}(t) \times$ $\times F_{n C} c_{n}(y) \int_{0}^{t} e_{n C}(-\tau) \int_{0}^{L_{x}} c_{n}(u) \int_{0}^{L_{y}} s_{n}(v) \int_{0}^{L_{z}} c_{n}(w) \frac{C_{00}^{\gamma}(u, v, w, \tau)}{P^{\gamma}(u, v, w, T)} \frac{\partial C_{01}(u, v, w, \tau)}{\partial v} d w d v d u d \tau \times$ $\times n c_{n}(z)-\frac{2 \pi}{L_{x} L_{y} L_{z}^{2}} \sum_{n=1}^{\infty} n F_{n C} c_{n}(x) c_{n}(y) c_{n}(z) e_{n C}(t) \int_{0}^{t} e_{n C}(-\tau) \int_{0}^{L_{x}} c_{n}(u) \int_{0}^{L_{y}} c_{n}(v) \int_{0}^{L_{z}} s_{n}(w) \times$ $\times \frac{C_{00}^{\gamma}(u, v, w, \tau)}{P^{\gamma}(u, v, w, T)} \frac{\partial C_{01}(u, v, w, \tau)}{\partial w} d w d v d u d \tau$

$C_{11}(x, y, z, t)=-\frac{2 \pi}{L_{x}^{2} L_{y} L_{z}} \sum_{n=1}^{\infty} n F_{n C} c_{n}(x) c_{n}(y) c_{n}(z) e_{n C}(t) \int_{0}^{t} e_{n C}(-\tau) \int_{0}^{L_{x}} S_{n}(u) \int_{0}^{L_{y}} c_{n}(v) \int_{0}^{L_{z}} c_{n}(w) \times$ $\times g_{L}(u, v, w, T) \frac{\partial C_{01}(u, v, w, \tau)}{\partial u} d w d v d u d \tau-\frac{2 \pi}{L_{x} L_{y}^{2} L_{z}} \sum_{n=1}^{\infty} n F_{n C} c_{n}(x) c_{n}(y) c_{n}(z) e_{n C}(t) \times$ $\times \int_{0}^{t} e_{n C}(-\tau) \int_{0}^{L_{x}} c_{n}(u) \int_{0}^{L_{y}} S_{n}(v) \int_{0}^{L_{z}} c_{n}(w) g_{L}(u, v, w, T) \frac{\partial C_{01}(u, v, w, \tau)}{\partial v} d w d v d u d \tau-\frac{2 \pi}{L_{x} L_{y} L_{z}^{2}} \times$ 


$$
\begin{aligned}
& \times \sum_{n=1}^{\infty} n e_{n C}(t) \int_{0}^{t} e_{n C}(-\tau) \int_{0}^{L_{x}} c_{n}(u) \int_{0}^{L_{y}} c_{n}(v) \int_{0}^{L_{z}} S_{n}(w) g_{L}(u, v, w, T) \frac{\partial C_{01}(u, v, w, \tau)}{\partial w} d w d v d u d \tau \times \\
& \times F_{n C} c_{n}(x) c_{n}(y) c_{n}(z)-\frac{2 \pi}{L_{x}^{2} L_{y} L_{z}} \sum_{n=1}^{\infty} F_{n C} c_{n}(x) c_{n}(y) c_{n}(z) e_{n C}(t) \int_{0}^{t} e_{n C}(-\tau) \int_{0}^{L_{x}} S_{n}(u) \int_{0}^{L_{y}} c_{n}(v) \times \\
& \times n \int_{0}^{L_{z}} c_{n}(w) \frac{C_{00}^{\gamma}(u, v, w, \tau)}{P^{\gamma}(u, v, w, T)} \frac{\partial C_{10}(u, v, w, \tau)}{\partial u} d w d v d u d \tau-\frac{2 \pi}{L_{x} L_{y}^{2} L_{z}} \sum_{n=1}^{\infty} n F_{n C} c_{n}(x) c_{n}(y) \times \\
& \times c_{n}(z) e_{n C}(t) \int_{0}^{t} e_{n C}(-\tau) \int_{0}^{L_{x}} c_{n}(u) \int_{0}^{L_{y}} s_{n}(v) \int_{0}^{L_{z}} c_{n}(w) \frac{C_{00}^{\gamma}(u, v, w, \tau)}{P^{\gamma}(u, v, w, T)} \frac{\partial C_{10}(u, v, w, \tau)}{\partial v} d w d v d u d \tau- \\
& -\frac{2 \pi}{L_{x} L_{y} L_{z}^{2}} \sum_{n=1}^{\infty} n F_{n C} c_{n}(x) c_{n}(y) c_{n}(z) e_{n C}(t) \int_{0}^{t} e_{n C}(-\tau) \int_{0}^{L_{x}} c_{n}(u) \int_{0}^{L_{y}} c_{n}(v) \int_{0}^{L_{z}} S_{n}(w) \frac{C_{00}^{\gamma}(u, v, w, \tau)}{P^{\gamma}(u, v, w, T)} \times \\
& \times \frac{\partial C_{10}(u, v, w, \tau)}{\partial w} d w d v d u d \tau-\frac{2 \pi}{L_{x}^{2} L_{y} L_{z}} \sum_{n=1}^{\infty} n F_{n C} c_{n}(x) c_{n}(y) c_{n}(z) e_{n C}(t) \int_{0}^{t} e_{n C}(-\tau) \int_{0}^{L_{x}} s_{n}(u) \times \\
& \times \int_{0}^{L_{y}} c_{n}(v) \int_{0}^{L_{z}} c_{n}(w) C_{10}(u, v, w, \tau) \frac{C_{00}^{\gamma-1}(u, v, w, \tau)}{P^{\gamma}(u, v, w, T)} \frac{\partial C_{00}(u, v, w, \tau)}{\partial u} d w d v d u d \tau-\frac{2 \pi}{L_{x} L_{y}^{2} L_{z}} \sum_{n=1}^{\infty} n \times \\
& \times F_{n C} c_{n}(x) c_{n}(y) c_{n}(z) e_{n C}(t) \int_{0}^{t} e_{n C}(-\tau) \int_{0}^{L_{x}} c_{n}(u) \int_{0}^{L_{y}} s_{n}(v) \int_{0}^{L_{z}} c_{n}(w) \frac{C_{00}^{\gamma-1}(u, v, w, \tau)}{P^{\gamma}(u, v, w, T)} \frac{\partial C_{00}(u, v, w, \tau)}{\partial v} \times \\
& \times C_{10}(u, v, w, \tau) d w d v d u d \tau-\frac{2 \pi}{L_{x} L_{y} L_{z}^{2}} \sum_{n=1}^{\infty} n F_{n C} c_{n}(x) c_{n}(y) c_{n}(z) e_{n C}(t) \int_{0}^{t} e_{n C}(-\tau) \int_{0}^{L_{x}} c_{n}(u) \times \\
& \times \int_{0}^{L_{y}} c_{n}(v) \int_{0}^{L_{z}} s_{n}(w) C_{10}(u, v, w, \tau) \frac{C_{00}^{\gamma-1}(u, v, w, \tau)}{P^{\gamma}(u, v, w, T)} \frac{\partial C_{00}(u, v, w, \tau)}{\partial w} d w d v d u d \tau .
\end{aligned}
$$

\section{REFERENCES}

[1] V.I. Lachin, N.S. Savelov. Electronics (Phoenix, Rostov-na-Donu, 2001).

[2] A.G. Alexenko, I.I. Shagurin. Microcircuitry (Radio and communication, Moscow, 1990).

[3] N.A. Avaev, Yu.E. Naumov, V.T. Frolkin. Basis of microelectronics (Radio and communication, Moscow, 1991).

[4] Z. Wang, Q. Duan, J. Roh. 2014. A $0.03 \mathrm{~mm} 2$ delta-sigma modulator with cascaded-inverter amplifier. Analog. Integr. Circ. Sig. Process. Vol. 81. P. 495-501 (2014). DOI 10.1007/s10470-014-0408-8.

[5] D. Fathi, B. Forouzandeh, N. Masoumi. 2009. New enhanced noise analysis in active mixers in nanoscale technologies. Nano. Vol. 4 (4). P. 233-238. https://doi.org/ 10.1142/S 1793292009001708 .

[6] S.A. Chachuli, P.N.A. Fasyar, N. Soin, N.M. Kar, N. Yusop. 2014. Pareto ANOVA analysis for CMOS $0.18 \mu \mathrm{m}$ two-stage Op-amp. Mat. Sci. Sem. Proc. Vol. 24. P. 9-14. https://doi.org/10.1016/j.mssp.2014.02.035.

[7] A.O. Ageev, A.E. Belyaev, N.S. Boltovets, V.N. Ivanov, R.V. Konakova, Ya.Ya. Kudrik, P.M. Litvin, V.V. Milenin, A.V. Sachenko. 2009. Au-TiBx-n-6H-SiC Schottky barrier diodes: Specific features of charge transport in rectifying and nonrectifying contacts. Semiconductors. Vol. 43 (7). P. 865-871. https://doi.org/ 10.1134/S1063782609070070.

[8] Z. Li, J. Waldron, T. Detchprohm, C. Wetzel, R. F. Karlicek Jr.,T. P. Chow. 2013. Monolithic integration of light-emitting diodes and power metal-oxide-semiconductor channel high-electron-mobility transistors for light-emitting power integrated circuits in GaN on sapphire substrate. Appl. Phys. Lett. Vol. 102 (19). P. 192107-192109. https://doi.org/10.1063/1.4807125 
[9] Jung-Hui Tsai, Shao-Yen Chiu, Wen-Shiung Lour, Der-Feng Guo. 2009. High-performance InGaP/GaAs pnp $\delta$-doped heterojunction bipolar transistor. Semiconductors. Vol. 43 (7). C. 939-942. https://doi.org/10.1134/S1063782 609070227.

[10] O.V. Alexandrov, A.O. Zakhar'in, N.A. Sobolev, E.I. Shek, M.M. Makoviychuk, E.O. Parshin. 1998. Formation of donor centers upon annealing of dysprosium-and holmium-implanted silicon. Semiconductors. Vol. 32 (9). P. 921-923. https://doi.org/10.1134/1.1187515.

[11] M.J. Kumar, T.V. Singh. 2008. Int. J. Nanoscience. Vol. 7 (2-3). P. 81-84.

[12] P. Sinsermsuksakul, K. Hartman, S.B. Kim, J. Heo, L. Sun, H.H. Park, R. Chakraborty, T. Buonassisi, R.G. Gordon. 2013. Enhancing the efficiency of $\mathrm{SnS}$ solar cells via band-offset engineering with a zinc oxysulfide buffer layer. Appl. Phys. Lett. Vol. 102 (5). P. 053901-053905. https://doi.org/10.1063/1.4789855.

[13] J.G. Reynolds, C.L. Reynolds, Jr.A. Mohanta, J.F. Muth, J.E. Rowe, H.O. Everitt, D.E. Aspnes. 2013. Shallow acceptor complexes in p-type ZnO. Appl. Phys. Lett. Vol. 102 (15). P. 152114-152118. https://doi.org/10.1063/1.4802753.

[14] K.K. Ong, K.L. Pey, P.S. Lee, A.T.S. Wee, X.C. Wang, Y.F. Chong. 2006. Dopant distribution in the recrystallization transient at the maximum melt depth induced by laser annealing. Appl. Phys. Lett. Vol. 89 (17). P. 172111-172114. https://doi.org/10.1063/1.2364834

[15] H.T. Wang, L.S. Tan, E.F. Chor. 2005. Pulsed laser annealing of Be-implanted GaN J. Appl. Phys. Vol. 98 (9). P. 094901-094905. https://doi.org/10.1063/ 1.2120893.

[16] S.T. Shishiyanu, T.S. Shishiyanu, S.K. Railyan. 2002. Shallow $\mathrm{p}-\mathrm{n}$ junctions formed in silicon using pulsed photon annealing. Semiconductors. Vol. 36 (5). P. 581-587. DOI https://doi.org/10.1134/1.1478552.

[17] Yu.V. Bykov, A.G. Yeremeev, N.A. Zharova, I.V. Plotnikov, K.I. Rybakov, M.N. Drozdov, Yu.N. Drozdov, V.D. Skupov. 2003. Diffusion Processes in Semiconductor Structures During Microwave Annealing. Radiophysics and Quantum Electronics. Vol. 46 (3). P. 749-755. https://doi.org/10.1023/B:RAQE. 0000025008.97954.1c

[18] E.L. Pankratov, E.A. Bulaeva. 2013. Doping of materials during manufacture $\mathrm{p}$-n-junctions and bipolar transistors. Analytical approaches to model technological approaches and ways of optimization of distributions of dopants. Reviews in Theoretical Science. Vol. 1 (1). P. 58-82. https://doi.org/10.1166/rits.2013.1004.

[19] Yu.N. Erofeev. Pulse devices (Higher School, Moscow, 1989).

[20] V.V. Kozlivsky. Modification of semiconductors by proton beams (Nauka, Sant-Peterburg, 2003).

[21] Z.Yu. Gotra. Technology of microelectronic devices (Radio and communication, Moscow, 1991).

[22] V.L. Vinetskiy, G.A. Kholodar', Radiative physics of semiconductors. ("Naukova Dumka", Kiev, 1979).

[23] P.M. Fahey, P.B. Griffin, J.D. Plummer. 1989. Diffusion and point defects in silicon. Rev. Mod. Phys. Vol. 61 (2). P. 289-388. DOI: https://doi.org/10.1103/RevModPhys.61.28
9.

[24] A.N. Tikhonov, A.A. Samarskii. The mathematical physics equations (Moscow, Nauka 1972).

[25] H.S. Carslaw, J.C. Jaeger. Conduction of heat in solids (Oxford University Press, 1964).

[26] E.L. Pankratov. 2007. Dopant diffusion dynamics and optimal diffusion time as influenced by diffusion-coefficient nonuniformity. Russian Microelectronics. Vol. 36 (1). P. 33-39.https://doi.org/10.1 134/S1063739707010040.

[27] E.L. Pankratov. 2008. Redistribution of a dopant during annealing of radiation defects in a multilayer structure by laser scans for production of an implanted-junction rectifier. Int. J. Nanoscience. Vol. 7 (4-5). P. 187-197 (2008). https://doi.org/10.1142/S0219581X08005328.

[28] E.L. Pankratov. 2017. On approach to optimize manufacturing of bipolar heterotransistors framework circuit of an operational amplifier to increase their integration rate. Influence Mismatch-Induced Stress J. Comp. Theor. Nanoscience. Vol. 14 (10). P. 4885-4899. https://doi.org/10.1166/jctn.2017.6899.

[29] E.L. Pankratov. 2017. On optimization of manufacturing of two-phase logic circuit based on heterostructures to increase density of their elements. Influence of miss-match induced stress Advanced science, engineering and medicine. Vol. 9 (9). P. 787-801. DOI: https://doi.org/10.1166/asem. 2017.2043.

[30] E.L. Pankratov, E.A. Bulaeva. 2017. On increasing of density of transistors in a hybrid cascaded multilevel inverter. Multidiscipline Modeling in Materials and Structures. Vol. 13 (4). P. 664-677. https://doi.org/10.1108 /MMMS-05-2017-0041.

[31] E.L. Pankratov, E.A. Bulaeva. 2014. An approach to manufacture of bipolar transistors in thin film structures. On the method of optimization. Int. J. Micro-Nano Scale Transp. Vol. 4 (1). P. 17-31.

[32] E.L. Pankratov, E.A. Bulaeva. 2016. An analytical approach for analysis and optimization of formation of field-effect heterotransistors. Multidiscipline Modeling in Materials and Structures. Vol. 12 (4). P. 578-604.https://doi.org/10.1 108/ MMMS-09-2015-0057.

[33] E.L. Pankratov, E.A. Bulaeva. 2015. An approach to increase the integration rate of planar drift heterobipolar transistors. Materials science in semiconductor processing. Vol. 34. P. 260-268. http://dx.doi.org/10.1016/j.mssp.2015 $.02 .0541369-8001$. 